\title{
M-Theory with Framed Corners and Tertiary Index Invariants
}

\author{
Hisham SATI
}

Department of Mathematics, University of Pittsburgh, Pittsburgh, PA 15260, USA

E-mail: hsati@pitt.edu

Received March 19, 2013, in final form March 01, 2014; Published online March 14, 2014

http://dx.doi.org/10.3842/SIGMA.2014.024

\begin{abstract}
The study of the partition function in M-theory involves the use of index theory on a twelve-dimensional bounding manifold. In eleven dimensions, viewed as a boundary, this is given by secondary index invariants such as the Atiyah-Patodi-Singer eta-invariant, the Chern-Simons invariant, or the Adams e-invariant. If the eleven-dimensional manifold itself has a boundary, the resulting ten-dimensional manifold can be viewed as a codimension two corner. The partition function in this context has been studied by the author in relation to index theory for manifolds with corners, essentially on the product of two intervals. In this paper, we focus on the case of framed manifolds (which are automatically Spin) and provide a formulation of the refined partition function using a tertiary index invariant, namely the $f$-invariant introduced by Laures within elliptic cohomology. We describe the context globally, connecting the various spaces and theories around M-theory, and providing a physical realization and interpretation of some ingredients appearing in the constructions due to Bunke-Naumann and Bodecker. The formulation leads to a natural interpretation of anomalies using corners and uncovers some resulting constraints in the heterotic corner. The analysis for type IIA leads to a physical identification of various components of eta-forms appearing in the formula for the phase of the partition function.
\end{abstract}

Key words: anomalies; manifolds with corners; tertiary index invariants; M-theory; elliptic genera; partition functions; eta-forms

2010 Mathematics Subject Classification: 81T50; 55N20; 58J26; 58J22; 58J28; $81 \mathrm{~T} 30$

\section{Introduction}

The goal of this paper is to combine the appearance of corners with that of elliptic cohomology to describe global aspects of the partition function in M-theory, which we hope could help shed some light on the role of elliptic cohomology in physics. Topological study of M-theory is often facilitated by taking it as a boundary. Furthermore, the heterotic theory is essentially a boundary of M-theory. Then, considering topological aspects in this setting requires the study of a twelve-dimensional theory whose boundary theory itself admits a boundary, i.e. forms a corner of codimension two. The partition function using index theory for manifolds with corners is analyzed in [63]. On the other hand, the study of anomalies in M-theory and string theory suggests connections to elliptic cohomology. In the case of heterotic string theory this has a long history, in particular in connection to elliptic genera [37, 74, 85]. More recently, by interpreting various anomaly cancellation conditions as orientations with respect to generalized cohomology theories, direct connections between elliptic cohomology, on the one hand, and M-theory and type II string theory, on the other hand, are uncovered [39, 40, 41, 55, 60, 72].

It is natural to ask how the above two descriptions can be consistently merged together. We advocate that the combination of the two pictures, namely that of codimension two corners and that of elliptic cohomology, fits nicely into a coherent structure form the mathematical point of view. We implement a unified view which we use to study some topological aspects of M-theory in this context for framed manifolds. This is done via the $f$-invariant, a tertiary invariant 
introduced in [42] and connected to index theory in [19, 83]. Some aspects of M-theory on framed manifolds in the context of elliptic cohomology are considered in [61]. Here, in addition to extending the relation further to the heterotic theory, we also consider the effective action and partition function of type IIA string theory.

Why framed manifolds. We would like to encode corners together with cobordism in the context of M-theory. By now it is established that cobordism invariants corresponding to various structures appear in the construction of the partition function in M-theory [21, 22, 56, 61, 68]. Structures described previously include Spin, Spin ${ }^{c}$ and String structures. These are related to K-theory and elliptic cohomology. In this paper we will consider manifolds with a more basic structure, namely a framing of the tangent bundle, which include the class of parallellizable manifolds. By the Pontrjagin-Thom construction, this amounts to dealing with the sphere spectrum, which in turn can be studied by means of elliptic cohomology. We, therefore, provide another angle on the proposals in [39, 40, 41, 60]. On the other hand, Lie groups provide an interesting class of framed manifolds, and so taking spacetime to be a Lie group (or some quotient thereof) resembles - and to some extent subsumes - Wess-Zumino-Witten models. Note, however, that being framed automatically means being Spin, so that our discussion certainly includes the structures that are expected from a physics point of view, namely Spin structures (see [68] for an extensive description, with an emphasis on the geometry).

Why framed in the heterotic theory. We would like to concentrate on the case when the 10-dimensional heterotic corner of M-theory admits a framing. String theory on parallelizable backgrounds is exactly solvable, and hence such backgrounds play a prominent role in the theory. In [26, 30, 34, 54] a classification of (simply-connected) supersymmetric parallelizable backgrounds of heterotic string theory is given. For heterotic backgrounds without gauge fields the dilaton is linear and hence can be described by a Liouville theory, and the geometry is that of a parallelized Lie group and hence can be described by a WZW model. These include products of Minkowski spaces with the odd-dimensional spheres $S^{3}$ and $S^{7}$ and the Lie group SU(3). For example, for the latter, the ten-dimensional manifold is $\mathbb{R}^{1,1} \times \mathrm{SU}(3)$. In the presence of nonzero gauge field strength, the geometry may be deformed away from that of a group manifold; it is still parallelizable but with respect to a metric connection with a skew-symmetric torsion [29]. Flux compactifications on group manifolds in heterotic string theory are considered in [11]. These include the group manifolds with zero Euler characteristic underlying ungauged WZW models, such as $S^{3} \times S^{1}$ [79]. Examples with nonozero Euler characteristic include connected sums of $\mathrm{SU}(2) \times \mathrm{SU}(2) \cong S^{3} \times S^{3}$, which admit a complex structure, but are non-Kähler, and have a nowhere-zero holomorphic form.

Index-theoretic invariants in various dimensions. Various index-theoretic invariants appear in the description of the effective action, and hence of the partition function, in M-theory. These arise in the form of an index in the twelve-dimensional extension of M-theory, a mod 2 index in type IIA in ten dimensions, and a secondary invariant in eleven-dimensional M-theory. Our point of view provides and supports a dimensional hierarchy of the form

- Dimension 4 and 12: The effective action is given by indices of twisted Dirac operator (see [86]). This is a main point where topology enters. Some refinements to elliptic genera appear in [39,61].

- Dimension 3 and 11: The effective action involves the eta-invariant, the $e$-invariant, or the Chern-Simons invariant (see [68] for an extensive discussion). In the presence of corners, the Melrose $b$-calculus is used to replace the eta-invariant with the $b$-eta-invariant [63]. Some elliptic refinements, in the sense of $[18,28]$, are discussed in [61].

- Dimension 2 and 10: The effective action and partition function of type II target string theory and of the worldsheet theory involve the Arf invariant [22]. Elliptic refinements of the mod 2 index include the Ochanine invariant as discussed in detail in [57]. 
Including the case of framed manifolds, we will advocate the structures in the following table

\begin{tabular}{|c|c|c|l|l|}
\hline Dimension & Physical Theory & Index invariant & Cohomology Theory & Underlying Structure \\
\hline \hline 10 & IIA & $d$ & KO-theory & Closed Spin manifold \\
\hline 11 & M-theory & $e$ & K-theory & Manifold with boundary \\
\hline 10 & Heterotic & $f$ & Elliptic cohomology & Manifold with codim-2 corner \\
\hline
\end{tabular}

Here $d$ is the Adams $d$-invariant, which is the degree (Hurewicz map) for KO-theory and is (a variant of) the mod 2 index of the Dirac operator ${ }^{1}, e$ is the Adams $e$-invariant, and $f$ is the invariant of Laures for manifolds with corners of codimension two in the context of elliptic cohomology. The latter is related to the elliptic genus in a manner similar to how the $e$-invariant is related to the Todd genus in K-theory. In the generalization of the $e$-invariant, which takes values in $\mathbb{Q} / \mathbb{Z}$, to modular forms one notes the following: Since $\mathbb{Q} / \mathbb{Z}$ is not a ring then it does not make sense to consider "modular forms with coefficients mod $\mathbb{Z}$ ". Hence, one has to consider modular forms with values in an appropriate ring, which turns out to be the ring of divided congruences $D$ [42]. More precisely, unlike the 1-line in the Adams-Novikov spectral sequence (ANSS) the 2-line is not cyclic in each dimension, hence one needs more copies of $\mathbb{Q} / \mathbb{Z}$, and a good way to do so is via $D \otimes \mathbb{Q} / \mathbb{Z}$.

Which elliptic cohomology theory? Topological modular forms (TMF), while can be viewed as a sort of a 'universal elliptic cohomology theory', suffers from a shortcoming, namely that it is not complex-oriented. The latter is desirable when dealing with physics (see [39, 40]). Therefore, we will consider versions of TMF which are complex-oriented. A prominent example is $\operatorname{TMF}_{1}(N)$ attached to the universal curve over the ring of integral modular forms for the congruence subgroup $\Gamma=\Gamma_{1}(N)$ of $\operatorname{SL}(2, \mathbb{Z})$. More precisely, $\operatorname{TMF}_{1}(N)$ is formed of global sections of a sheaf of spectra over the moduli space of elliptic curves with level structure; see $[42,46]$. Although elliptic cohomology of level 2 allows to extract the information on the $e$-invariant, its use is by no means a requirement as, in fact, KO-theory is enough for that purpose. The important point is that $\mathrm{TMF}_{1}(N)$ provides natural refinements of "well-known" invariants, such as $d$ and $e$, as well as entirely new ones, such as $f$. An appropriate value for $N$ turns out to be 3 . In K-theory at times one works away from powers of 2 , and the analogy here is working in elliptic cohomology away from powers of 3 . In fact, if one does not want 2 to be inverted then the smallest level at which this occurs is 3 . Detecting mod 3 phenomena, i.e. considering the case $N=3$, connects to anomalies at the prime 3 , studied in $[25,56]$. The congruence subgroup $\Gamma_{1}(3)$ appears elsewhere physics, e.g. in the context of topological string theory [1]. Note, however, that one should exercise caution in that working at a fixed prime level is not equivalent to focusing on phenomena (e.g. anomalies) at that prime.

Elliptic genera for the heterotic string. We recall an explicit instance where elliptic genera appear in the heterotic theory, which we will later view as a corner. The modular invariance violating terms can be factored out of the character-valued partition function, which has the form [44]

$$
A(q, F, R)=\exp \left[-\frac{64}{\pi^{4}} G_{2}(\tau)\left(\operatorname{Tr} F^{2}-\operatorname{Tr} R^{2}\right)\right] \widetilde{A}(q, F, R),
$$

where $\widetilde{A}(q, F, R)$ is a fully holomorphic and modular invariant of weight -4 , and can be expressed in terms of the Eisenstein functions $G_{4}$ and $G_{6}$ (or equivalently, using $E_{4}$ and $E_{6}$ ), as opposed

\footnotetext{
${ }^{1}$ Note that Adams defined the degree $d$ for any (generalized) cohomology theory. It is the one based on KOtheory which can be interpreted as an index $\bmod 2$. On the other hand, the one based on integral cohomology $H \mathbb{Z}$ is the 'correct' one from the chromatic point of view (i.e. it detects 0th filtration phenomena), but does not carry 'nontrivial' information.
} 
to the function $G_{2}$ which is not modular. From modular invariance, the anomaly always has a factorized form and is given by the constant term in the elliptic genus

$$
I_{12}(F, R)=\left.A(q, F, R)\right|_{12 \text {-form coeff. of } q^{0}}=\frac{1}{2 \pi}\left(\operatorname{Tr} F^{2}-\operatorname{Tr} R^{2}\right) \wedge X_{8}(F, R),
$$

where $X_{8}(F, R)$ is the Green-Schwarz polynomial corresponding to the tangent bundle and gauge bundle with curvatures $R$ and $F$, respectively.

Oultine. What we do in this paper can be summarized in the following:

1. We provide a setting for framed manifolds in M-theory and string theory, starting at the beginning of Section 2. We specialize to parallelizable manifolds, stably parallelizable manifolds, and in particular to Lie groups and homogeneous spaces in Sections 2.1, 2.2, and 2.3, respectively. Since M-theory involves boundaries, we describe how framed manifolds arise as boundaries in our context in Section 2.4.

2. We describe framed cobordism invariants in connection to M-theory and string theory. After a general discussion on framed cobordism in Section 2.5, we describe the relation between 10-dimensional string theory and the $d$-invariant (which is a variant of the Arf invariant) in Section 2.6.1, and then the relation between the $e$-invariant and 11-dimensional M-theory in Section 2.6.2. Along the way we explain the effect on the partition function, and in Section 2.7 we consider that from the point of view of change of framing. We also describe the parity symmetry of the $C$-field in this context.

3. Having described both framed structures and invariants, we introduce the corner formulation and show in Section 3.1 how the various interconnected theories, together with their boundaries and some dualities, fit nicely into the structure of framing and corners. This provides a transparent view on how K-theory and elliptic cohomology enter into the setting. For instance, the formulation of the $C$-field in M-theory in [21] can be cast in this setting in a very natural way.

4. We consider type IIA string theory on a manifold with boundary in connection to M-theory, itself with a boundary and as a boundary. Since the phase of the partition function is given by an index, it is natural to describe the partition function, and especially the phase, in this indextheoretic context. This allows us to get an expression of the phase and provide an interpretation of eta-forms appearing in [48], thereby extending similar interpretations in [59,63]. This also serves as a warm up via secondary index theory for the application of the tertiary index theory in later sections.

5. We consider the formulation of the heterotic theory as a corner in Section 3.3. The factorization of the anomaly can be viewed via the splitting of the tangent bundle in the context of framing. Furthermore, we show in Section 3.3.1 that the general form of the anomaly as well as the cancellation of that anomaly point to the presence of corners. This can be generalized to anomaly cancellation as a general process. Then, in Section 3.3.2, we lift the one-loop term to twelve dimensions and then consider the reduction to the corner. This results in a constraint on degree twelve Chern numbers that generalize the constraint on degree ten Chern numbers in [22], and gives rise to a cup product composite Chern-Simons theory. We illustrate how these conditions affect the corner by highlighting the example of the ten-dimensional Lie group $\operatorname{Sp}(2)$ in [42] which turns out to be the physically important one (see [25]).

6. We next combine the framing and corners description of the heterotic string with the elliptic cohomology aspect via the framed cobordism invariant at chromatic level 2 , namely the $f$ invariant of Laures [42], and its geometric refinement by Bunke-Naumann [19] and Bodecker [83]. This can be viewed as the reduction of the index - i.e. the topological part of the action - from twelve dimensions to the corner. In Section 3.4 we highlight the connection to topological modular forms and Tate K-theory and how the terms in the effective action get refined to $q$ expansions, as in [61]. As we explain in Section 3.5, the $f$-invariant captures the nonzero $q$ part 
of the expansion and hence, in the view of treating $q$ as a sort of a 'coupling constant' [55], the quantum aspects of the theory. We highlight this in the example of $S^{3} \times S^{7}$.

We emphasize exposition to explain the various interconnections between the mathematical constructions and the physical ingredients and settings.

\section{Framed manifolds and framed bundles in M-theory}

In this section we describe relevant classes of framed manifolds which appear in our setting. These include parallelizable manifolds such as Lie groups and certain homogeneous spaces. We then highlight the relevance of structures on these spaces to the physics in M-theory and string theory. We will denote by "admissible manifolds" those manifolds that can be taken as spaces on which string theory or M-theory can be compactified, with or without fluxes.

Framed manifolds. If $M$ is a closed $n$-dimensional manifold with tangent bundle $T M$, then its stable tangent bundle ${ }^{2} T^{\text {st }} M$ is the direct sum of $T M$ with a large ${ }^{3}$ trivial bundle $M \times \mathbb{R}^{r}$

$$
T^{\mathrm{st}} M \cong T M \oplus\left(M \times \mathbb{R}^{r}\right)
$$

A (stable) framing on $M$ means a trivialization $f$ of $T^{\text {st }} M$, that is, a set $f=\left(f_{1}, \ldots, f_{n+r}\right)$ of $(n+r)$-sections of $T^{\text {st }} M$, linearly independent everywhere. One can consider this from the point of view of embeddings. A framing on a manifold $M^{n}$ smoothly embedded in Euclidean space $\mathbb{R}^{n+k}$ consists of an ordered set of vectors $\left\{v_{1}(x), \ldots, v_{k}(x)\right\}$ varying smoothly with $x \in M^{n}$ and providing a basis for the normal space of $M^{n}$ in $\mathbb{R}^{n+k}$ at $x$. In terms of classifying spaces of $G$-structures (see [81]), a framing on a smooth manifold $M$ is a pair $(h, \tilde{\nu})$ such that $h: M \rightarrow$ $\mathbb{R}^{n+k}$ is an embedding with normal bundle classified by a map $\nu: M \rightarrow \mathrm{BO}(k)$ with a lifting $\tilde{\nu}: M \rightarrow \mathrm{EO}(k)$, where $\mathrm{EO}(k)$ is the total space of the universal principal $\mathrm{O}(k)$ bundle

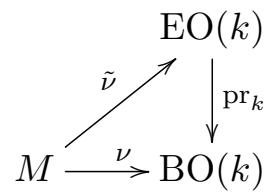

with $\operatorname{pr}_{k}: \mathrm{EO}(k) \rightarrow \mathrm{BO}(k)$ being the bundle projection.

Framed vector bundles. A rank $r$ vector bundle $E \rightarrow M$ is called trivial or trivializable if there exists a bundle isomorphism $E \cong M \times \mathbb{R}^{r}$ with the trivial rank $r$ bundle over $M$. A bundle isomorphism $E \rightarrow M \times \mathbb{R}^{r}$ is called a trivialization of $E$, while an isomorphism $\varphi: M \times \mathbb{R}^{r} \rightarrow E$ is called a framing of $E$. Denote by $\left(e_{1}, \ldots, e_{r}\right)$ the canonical basis of the vector space $\mathbb{R}^{r}$, and regard the vectors $e_{i}$ as constant maps $M \rightarrow \mathbb{R}^{r}$, i.e. as sections of $M \times \mathbb{R}^{r}$. The isomorphism $\varphi$ determines sections $f_{i}=\varphi\left(e_{i}\right)$ of $E$ with the property that for every $x \in M$ the collection $\left(f_{1}(x), \ldots, f_{r}(x)\right)$ is a frame of the fiber $E_{x}$. This shows that we can regard any framing of a bundle $E \rightarrow M$ of rank $r$ as a collection of $r$ sections $\left\{u_{1}, \ldots, u_{r}\right\}$ which are pointwise linearly independent. Thus one has that a pair "(trivial bundle, trivialization)" deserves to be called a trivialized, or framed bundle.

In the following we provide what might be viewed as a toolkit for admissible manifolds, whereby we provide an extensive class of examples.

\footnotetext{
${ }^{2}$ Note that this definition involves (commonly accepted) abuse of notation. Strictly speaking, the stable tangent bundle of $M$ should be thought of as an equivalence class of such, namely as a class in reduced real K-theory $\widehat{\mathrm{KO}}(M)$. A similar remark holds for the stable framing.

${ }^{3}$ That is, large $r$. However, the exact value of $r$ will not be important for us.
} 


\subsection{Parallelizable manifolds}

A parallelizable manifold is a manifold whose tangent bundle is trivial. A trivialization provides a framing in a natural way. It is important to emphasize that, in general, a trivial bundle is not canonically trivialized, a fact which is the source of anomalies from the geometric point of view.

As every parallelizable manifold is Spin, it is an admissible manifold (in a strong sense) in M-theory. Such manifolds are often decomposable. The product of two parallelizable manifolds is also parallelizable. However, the product of two stably parallelizable manifolds is not necessarily parallelizable. However, a product $M \times N$ is parallelizable if and only if $M$ and $N$ are stably parallelizable and either factor has a vanishing Euler characteristic, since $\chi(M \times N)=\chi(M) \cdot \chi(N)$. This is automatically satisfied if the total dimension is odd.

Examples. 1. Lie groups. All Lie groups (and their quotients by finite subgroups) are parallelizable. We discuss this important class of examples in detail in Section 2.3.

2. (Projective) Stiefel manifolds. The real and complex Stiefel manifolds $V_{n, k}$ are parallelizable if $k \geq 2$. For complex Stiefel manifolds one has the following (see [3]). $P V_{n, k}$ is the quotient space of the free circle action on the complex Stiefel manifold $V_{n, k}$ of orthonormal $k$-frames in complex $n$-space given by $z\left(v_{1}, \ldots, v_{k}\right)=\left(z v_{1}, \ldots, z v_{k}\right)$. If $k<n-1$ then $P V_{n, k}$ is not stably parallelizable. The manifold $P V_{n, n-1}$ is parallelizable, except $P V_{2,1}=S^{2}$, while $P V_{n, n}$ is the projective unitary group, and so is parallelizable.

3. Grassmannian manifolds. The only real Grassmannian manifolds $\operatorname{Gr}_{k}\left(\mathbb{R}^{n}\right)$ which are parallelizable are the obvious cases: $\operatorname{Gr}_{1}\left(\mathbb{R}^{2}\right) \cong \mathbb{R} P^{1}, \mathrm{Gr}_{1}\left(\mathbb{R}^{4}\right) \cong \mathrm{Gr}_{3}\left(\mathbb{R}^{4}\right) \cong \mathbb{R} P^{3}$ and $\mathrm{Gr}_{1}\left(\mathbb{R}^{8}\right) \cong$ $\operatorname{Gr}_{7}\left(\mathbb{R}^{8}\right) \cong \mathbb{R} P^{7}$. The ten-dimensional manifold $X^{10}=\mathbb{R} P^{3} \times \mathbb{R} P^{7}$ plays an important role in the subtle aspects of K-theoretic description of the fields in type II string theory [15].

4. Homogeneous spaces. Many homogeneous spaces are known to be parallelizable: Lie groups, Stiefel manifolds, quotients of the form $G / T$ where $G$ is a Lie group and $T$ is a nonmaximal toral subgroup. This provides many examples involving the relevant low-rank Lie groups. Another relevant class of homogeneous spaces is the following. Let $G=\mathrm{SU}(n)$ and $H=$ $\mathrm{SU}\left(k_{1}\right) \times \cdots \times \mathrm{SU}\left(k_{r}\right), r=\operatorname{rank}(G)$, embedded in $G$ in an arbitrary manner. For an appropriate choice of $\left\{k_{i}\right\}$, if $G / H$ is parallelizable then $G / H$ is either a complex Stiefel manifold or is of the form $\mathrm{SU}(n) / \mathrm{SU}(2)^{\times k}$ where the subgroup $\mathrm{SU}(2)^{\times k}, 2 k \leq n$ is embedded in the standard fashion [75]. Most relevant for us is the nine-dimensional manifold $\mathrm{SU}(4) /(\mathrm{SU}(2) \times \mathrm{SU}(2))$.

5. Products of spheres. The product of a sphere with a sphere of odd dimensions is always parallelizable (see [35]). For example, let us consider the eleven-dimensional manifold $Y^{11}=S^{4} \times S^{7}$, which is important in the flux compactification of M-theory. The 7 -sphere $S^{7}$ admits a nowhere zero section, so that the tangent bundle is the sum $T S^{7}=\eta \oplus \varepsilon^{1}$ for some rank 6 bundle $\eta$. Let $\mathrm{pr}_{1}$ and $\mathrm{pr}_{2}$ denote the projections of the product to the first and second factors, respectively. Then $T\left(S^{4} \times S^{7}\right)=\operatorname{pr}_{1}^{*}\left(T S^{4}\right) \oplus \operatorname{pr}_{2}^{*}\left(\eta \oplus \varepsilon^{1}\right)$; now the second summand gives $\operatorname{pr}_{2}^{*}(\eta) \oplus \varepsilon^{1}$ and so using $\operatorname{pr}_{1}^{*}\left(T S^{4}\right) \oplus \varepsilon^{1}=\operatorname{pr}_{1}^{*}\left(T S^{4} \oplus \varepsilon^{1}\right)=\varepsilon^{5}$, in total we have $\operatorname{pr}_{2}^{*}\left(\eta \oplus \varepsilon^{5}\right)=\varepsilon^{11}$, which shows that indeed $S^{4} \times S^{7}$ is parallelizable. Similar remarks hold for the decomposable eleven-dimensional manifolds $S^{8} \times S^{3}, S^{6} \times S^{5}, S^{2} \times S^{9}$, and $S^{1} \times S^{10}$.

6. Principal bundles over parallelizable manifolds. If the base space of a principal bundle is parallelizable then so is the total space, since the fiber is isomorphic to a Lie group, which is parallelizable. In relating M-theory in eleven dimensions to various favors of string theory in lower dimensions, one performs dimensional reduction, which can usually be viewed as a reduction of the total space of a principal bundle to its base.

\subsection{Stably parallelizable manifolds}

A bundle $E$ is said to be stably trivial if its Whitney sum with a trivial bundle is trivial. A manifold $M$ is said to be stably parallelizable or a $\pi$-manifold if the tangent bundle $T M$ is 
stably trivial. Note that if $T M \oplus \varepsilon^{k}$ is trivial then $T M \oplus \varepsilon^{1}$ is already trivial. If a connected, stably parallelizable manifold $M$ has non-empty boundary, then it is actually parallelizable [36].

Reduction of structure group for stably parallelizable manifolds. Let $Y^{11}$ be a connected stably parallelizable closed eleven-dimensional manifold. There is, up to isomorphism, exactly one stably trivial, but not trivial, 11-dimensional vector bundle $\tau$ over $Y^{11}$. It may be described as the pullback of the tangent bundle of $S^{11}$ by a map $f: Y^{11} \rightarrow S^{11}$ of degree one (collapsing the complement of an open disk) [20]. It follows from [82] that the structure group of $\tau$ can be reduced to $\mathrm{SO}(k)$ by the standard inclusion $\mathrm{SO}(k) \hookrightarrow \mathrm{SO}(11)$ if and only if $12 \equiv 0$ mod $a(12-k)$, where $a(r)$ is the Hurwitz-Radon number of $r$. The special case in which $\tau$ is the tangent bundle is considered in [17].

Properties. (Stably) parallelizable manifolds enjoy the following useful properties.

1. The boundary of a parallelizable manifold is a $\pi$-manifold. This will be useful when considering various boundaries and corners.

2. The product of two $\pi$-manifolds is a $\pi$-manifold. This will be useful when we consider decomposable $\pi$-manifolds, which will be the main class of admissible manifolds.

3. Every stably parallelizable manifold is Spin. This also follows from the more general fact that framed manifolds are Spin. Hence such manifolds are physically admissible.

4. Suppose $H \subset K \subset G$ is a sequence of closed Lie groups. If $G / H$ is stably parallelizable then so is $K / H$.

Examples. 1. All parallelizable manifolds. This includes spheres in dimensions 1, 3, and 7. On the other hand, the only real Grassmannian manifolds $G_{k}\left(\mathbb{R}^{n}\right)$ which are stably parallelizable are the parallelizable ones, as in Section 2.1 above.

2. Spheres. While spheres are stably parallelizable. They have many interesting properties including $T S^{n} \oplus \varepsilon^{1}=\varepsilon^{n+1}$.

3. Homogeneous spaces. An example which is not (strictly) parallelizable is the following. Let $G$ be a simple 1-connected compact Lie group and $H$ a closed connected subgroup. Then $G / H$ is stably parallelizable if and only if the adjoint representation $\operatorname{Ad}_{H}$ of $H$ is contained in the image of the restriction map of real representation rings $\mathrm{RO}(G) \rightarrow \mathrm{RO}(H)$ [77]. A homogeneous space which is almost parallelizable not strictly parallelizable is $G / T_{\max }$, the quotient of a Lie group $G$ by a maximal torus.

4. Sphere bundles over $\pi$-manifolds. Recall that the tangent bundle of a sphere bundle $S(E)$ takes the form $T S(E) \cong \pi^{*} T M^{n} \oplus T_{F}(S(E))$, with the canonical isomorphism $1 \oplus$ $T_{F}(S(E)) \cong \pi^{*} E$, where $T_{F}$ is the vertical tangent bundle. Assume that $M^{n}$ is a $\pi$-manifold. Then $S(E)$ is a $\pi$-manifold if $\pi^{*} E \rightarrow S(E)$ is stably trivial. In particular, $S(E)$ is a $\pi$-manifold if $M^{n}$ is the $n$-sphere; see [78].

5. Sphere bundles over spheres. This is a class of examples that will be very useful for us when considering the partition function in later sections. Let $E \rightarrow M^{n}$ be a smooth oriented $m$ plane bundle with associated sphere bundle $S^{m-1} \rightarrow S(E) \rightarrow M^{n}$ in some Riemannian metric. We specify to $M^{n}=S^{n}$ and introduce the disk bundle $\mathbb{D}^{m} \rightarrow \mathbb{D}(E) \rightarrow S^{n}$ associated to a vector bundle $E$ over the sphere $S^{n}$. Recall that $T(\mathbb{D}(E)) \cong \pi^{*} T S^{n} \oplus \pi^{*} E$, so that if $\phi$ is a stable trivialization of $E$ there is induced a stable framing of $\mathbb{D}(E)$ by pulling back $\phi$ and the usual stable framing of $T S^{n}$ along $\pi$. Note that $\partial(\mathbb{D}(E) ; \phi)=(S(E) ; \phi)$ where $\phi$ is the stable framing of $S(E)$. In general, such bundles are only stably parallelizable.

Extending almost parallelizable to parallelizable manifolds. Here we will contrast the case of string theory and M-theory, in the sense of even- vs. odd-dimensional manifolds. If the dimension of $M$ is even, the parallelizability of a stably parallelizable manifold is determined by the vanishing of the Euler characteristic of $M$. Thus if the Euler characteristic is zero then any stably parallelizable manifold is in fact parallelizable. For us this includes ten-dimensional manifolds appearing in type IIA and heterotic string theory. On the other hand, if the dimension of $M$ is odd, $M$ is parallelizable if and only if its Kervaire semi-characteristic $\chi_{\frac{1}{2}}(M)$, defined 
via mod 2 homology by

$$
\chi_{\frac{1}{2}}(M)=\frac{1}{2} \sum_{i=0}^{\lfloor\operatorname{dim}(M) / 2\rfloor} \operatorname{dim}_{\mathbb{Z}_{2}} H_{i}\left(M ; \mathbb{Z}_{2}\right) \quad(\bmod 2)
$$

vanishes. Therefore, similarly, when $\chi_{\frac{1}{2}}(M)=0$ then a stably parallelizable manifold becomes parallelizable. This places a condition on the homology of the manifolds; see Section 3.1.

\subsection{Lie groups and homogeneous spaces as framed manifolds}

Lie groups form an interesting class of examples of compactification manifolds which are able to carry subtle torsion information about fields in spacetime. See e.g. [47] for a description of such WZW models in the context of twisted K-theory. The discussion we give below, together with the construction of twisted Morava K-theory in [73], allows for an extension to detect finer invariants (see Section 2.5).

Framings on Lie groups. The left invariant vector fields of a compact Lie group $G$ induce a specific isomorphism $\mathcal{L}$, the left invariant framing, between the tangent bundle of $G$ and the product bundle $G \times \mathbb{R}^{\operatorname{dim} G}$. Indeed, the tangent bundle $T(G)$ of any Lie group $G$ is trivial. Take a basis $\left\{e_{1}, \ldots, e_{n}\right\}$ of the tangent space at the origin $T_{e}(G)$, where $n=\operatorname{dim} G$. Denote by $R_{g}$ the right translation by $g$ in the group defined by $R_{g}: x \mapsto x \cdot g$, for all $x \in G$. This is a diffeomorphism with inverse $R_{g}^{-1}=R_{g^{-1}}$ so that the differential $D R_{g}$ defines a linear isomorphism $D R_{g}: T_{e}(G) \rightarrow T_{g} G$. Since the multiplication $G \times G \rightarrow G$, given by $(g, h) \mapsto g \cdot h$, is a smooth map then the vectors $f_{i}(g)=D R_{g}\left(e_{i}\right) \in T_{g} G, i=1, \ldots, n$, define smooth vector fields over $G$. Then for every $g \in G$ the set $\left\{f_{1}(g), \ldots, f_{n}(g)\right\}$ is a basis of $T_{g}(G)$ so that there is indeed a vector bundle isomorphism $\phi: G \times \mathbb{R}^{n} \rightarrow T G$ taking $\left(g ; e^{1}, \ldots, e^{n}\right)$ to $\left(g ; \sum e^{i} f_{i}(g)\right)$. Similarly, the same holds for framing via the left translation $\mathcal{L}_{g}$. Then the right invariant framing $\mathcal{R}: T(G) \cong G \times T_{e}(G)$ of $G$ is given by $\mathcal{R}(v)=\left(g, R_{g^{-1}}(v)\right)$ where $v \in T_{g}(G)$.

Framings on homogeneous spaces $\boldsymbol{G} / \boldsymbol{H}$. Let $G$ be a compact connected Lie group and $H$ a closed subgroup of $G$. Let $T(G / H)$ denote the tangent bundle bundle of the coset $G / H$. Consider the $H$-principal bundle $H \rightarrow G \stackrel{\pi}{\rightarrow} G / H$. Then the tangent bundle of $G$ decomposes as $T(G) \cong \pi^{*} T(G / H) \oplus T_{H}(G)$. This isomorphism is compatible with the right $H$-action, and so there is an isomorphism of vector bundles over $G / H$, namely $T(G) / H \cong T(G / H) \oplus$ $T_{H}(G) / H$. Let $\operatorname{ad}_{H}$ denote the adjoint representation of $H$ on $T_{e}(H)$. Similarly, there is an isomorphism $T_{H}(G) / H \cong G \times_{H} T_{e}(H)$ of vector bundles over $G / H$, where $H$ acts on $T_{e}(H)$ via $\operatorname{ad}_{H}$. Combining the above bundles gives the isomorphism of vector bundles over $G / H$

$$
G / H \times T_{e}(G) \cong T(G / H) \oplus G \times_{\operatorname{ad}_{H}} T_{e}(H) .
$$

So if $\operatorname{ad}_{H}$ is contained in the image of the restriction map $\mathrm{RO}(G) \rightarrow \mathrm{RO}(H)$ of real representation rings then (2.2) gives a framing of $G / H$ (see [45]).

\subsection{Framed boundaries}

We will consider framed manifolds in twelve, eleven, ten, and nine dimensions, and in the last three cases we would like to allow the manifolds to be boundaries. We will consider restrictions for this to occur and illustrate with useful examples.

Lie groups. Let $G$ be a compact Lie group. Two natural questions that arise in our context are: When is $G$ the boundary of a compact manifold $Z$ ? In this case, when is $Z$ parallelizable? We highlight two cases that are important to our discussion:

1. Disk bundles $\mathbb{D}\left(\mathcal{L}_{\mathbb{C}}\right)$ of the canonical complex line bundles $\mathcal{L}_{\mathbb{C}}$ over the quotient $G / S^{1}$. The boundary $Y$ of the total space $Z$ of the complex line bundle is the circle bundle $S(\mathcal{L})$ 
given by $G \rightarrow G / S^{1}$ with $\partial Z=G$. This context (for manifolds that are not necessarily framed) is discussed extensively in [68].

2. Similarly for disk bundles $\mathbb{D}\left(\mathcal{L}_{\mathbb{H}}\right)$ of the quaternionic line bundles $\mathcal{L}_{\mathbb{H}}$ over $G / \mathrm{SU}(2) \cong$ $G / S^{3}$. The boundary $Y$ of the total space $Z$ of the quaternionic line bundle is the sphere bundle $S\left(\mathcal{L}_{\mathbb{H}}\right)$ given by $G \rightarrow G / S^{3}$ with $\partial Z=G$. The example we have in mind in this case is the group ten-manifold $\operatorname{Sp}(2) \cong \operatorname{Spin}(5)$ or $\mathrm{SO}(5)$ and their quotients with finite groups. See also [25] for an application to D-brane anomalies.

Generalized flag manifolds. Let $G$ be a compact Lie group of rank $l$ and $T$ a maximal torus. Then the flag manifold $G / T$ is a $\pi$-manifold [14], and an explicit bounding manifold $W$ with a corresponding stable framing can be constructed as follows [53]. Let $\mathfrak{g}$ and $\mathfrak{t}$ be the Lie algebras of $G$ and $T$, respectively. Under the adjoint action of $T, \mathfrak{g}$ decomposes as an $\operatorname{ad}(T)$-module as $\mathfrak{g}=\mathfrak{t} \oplus_{\alpha} \mathfrak{g}_{\alpha}$, where $\alpha$ are certain linear forms $\alpha: \mathfrak{t} \rightarrow \mathbb{R}$ and the $\mathfrak{g}_{\alpha}$ are two-dimensional $T$-modules corresponding to $e^{\alpha}: T \rightarrow \operatorname{SO}(2)$. The subspace $\mathfrak{C}_{\alpha}=\mathfrak{t} \oplus \mathfrak{g}_{\alpha}$ is in fact a Lie subalgebra of $\mathfrak{g}$ isomorphic to $\mathbb{R}^{l-1} \oplus \mathfrak{s u}(2)$, where $\mathbb{R}^{l-1}=\operatorname{ker} \alpha$ is the center and $\mathfrak{s u}(2)$ is the commutator subalgebra of $\mathfrak{C}_{\alpha}$. Denote by $C_{\alpha} \leq G$ the closed connected subgroup corresponding to $\mathfrak{C}_{\alpha} \leq \mathfrak{g}$. Then the 2 -sphere bundle

$$
C_{\alpha} / T \rightarrow G / T \rightarrow G / C_{\alpha}
$$

has a corresponding disk bundle $W$ which is stably parallelizable. The tangent bundle of $W$ is given by $T W \cong \pi^{*} T\left(G / C_{\alpha}\right) \oplus \pi^{*} \xi$, where $\xi$ is the 3 -plane vector bundle corresponding to the 2 -sphere bundle (2.3). For example, when $G$ is the Lie group $G_{2}$ the total space of the 2-sphere bundle is a 12-dimensional manifold.

$\boldsymbol{S}^{\mathbf{1}}$ - and $\boldsymbol{S}^{\mathbf{3}}$-action and framing of the disk bundle. Consider the case when $H$ is $S^{1}$ or $S^{3}$ and $Z$ the corresponding disk bundle over $G / H$ with projection $p$. Denote by $\operatorname{Ad}_{G}$ $\left(\right.$ resp. $\left.\operatorname{Ad}_{H}\right)$ the adjoint representation of $G$ (resp. $H$ ). Then the restriction of $\operatorname{Ad}_{G}$ to $H$ decomposes as

$$
\left.\operatorname{Ad}_{G}\right|_{H}=\operatorname{Ad}_{(G, H)} \oplus \operatorname{Ad}_{H}
$$

since $\left.\operatorname{Ad}_{G}\right|_{H}$ contains $\operatorname{Ad}_{H}$ as a sub-representation. Let $H$ act via $\left.\operatorname{Ad}_{G}\right|_{H}$ on the tangent space $T_{e}(G)$, decomposing it via $(2.4)$, as $T_{e}(G)=V \oplus T_{e}(H)$. From $T(G) / H \cong G \times_{H} T_{e}(G)$ and $T_{H}(G) / H \cong G \times_{H} T_{e}(H)$, one gets $T(G / H) \cong G \times_{H} V$. Suppose that there is a real representation $f$ of $G$ such that $\left.f\right|_{H}=\operatorname{Ad}_{(G, H)} \oplus \sigma \oplus \ell$, where the integer $\ell$ denotes the $\ell$ dimensional trivial representation, and $\sigma$ is the inclusion $H \hookrightarrow \mathrm{SO}(r+1)$ for $r=1,3$. Applying $f$ to $T Z \cong p^{*}(T(G / H) \oplus \eta$, where $\eta$ is the vector bundle associated via $\sigma$ to the principal $H$-bundle $\pi: G \rightarrow G / H$, yields an isomorphism $\phi: T Z \oplus\left(Z \times \mathbb{R}^{\ell}\right) \rightarrow Z \times \mathbb{R}^{d+\ell+1}$, which provides a framing for $Z$. So the framed manifold $(Z, \phi)$ bounds the framed manifold $(G,-f)$. See [51] for more details. More examples can be found in [4].

We now present an example which is central to our discussion.

Example. Sp(2). The ten-dimensional Lie group $\mathrm{Sp}(2)$ can be viewed as a 3 -sphere bundle over the 7-sphere, $S^{3} \hookrightarrow \mathrm{Sp}(2) \rightarrow S^{7}$. This example is used in [25] to study D-brane anomalies at the prime $p=3$. Since this is a sphere bundle, it is a boundary of a disk bundle $\mathbb{D}^{4} \hookrightarrow Y^{11}=$ $D(\operatorname{Sp}(2)) \rightarrow S^{7}$, which is a Spin manifold. The framing can also be extended to the disk bundle by the above results, even though $[\operatorname{Sp}(2), \alpha, \mathcal{L}]=0$ in the stable homotopy group $\pi_{10}^{s} S^{0}$ (see Section 2.5).

Topological conditions on stably parallelizable manifolds with boundary. We consider M-theory on an eleven-manifold $Y^{11}$. Then the semi-characteristic of $Y$ is defined in (2.1). Let $Z^{12}$ be a compact 12 -dimensional manifold with boundary $\partial Z^{12}=Y^{11}$. Then, from the 
general result in [16], the Euler characteristic of $Z^{12}$ and the Euler semi-characteristic of $Y^{11}$ are related as

$$
\chi\left(Z^{12}\right)=\chi_{\frac{1}{2}}\left(Y^{11}\right) \bmod 2 .
$$

This places a condition on the cohomology of $Y^{11}$ and of its bounding manifold $Z^{12}$.

\subsection{Framed cobordism}

We are considering manifolds which can be boundaries and which at the same time admit a framing. The natural context to study these is framed cobordism.

Framed cobordism classes and the parity symmetry in M-theory. Let $M_{1}$ and $M_{2}$ be two closed $n$-dimensional framed manifolds. We say that $M_{1}$ and $M_{2}$ are framed cobordant (written $M_{1} \sim M_{2}$ ) if there are $(n+1)$-dimensional compact framed manifolds $W_{1}, W_{2}$ with framed diffeomorphism $M_{1} \amalg \partial W_{1} \cong M_{2} \amalg \partial W_{2}$, where $\partial W_{1}$ and $\partial W_{2}$ have the induced framings. The empty set can be viewed as an $n$-dimensional smooth framed manifold with a unique framing. A framed manifold $(M, f)$ is null-cobordant or cobordant to zero if $M$ is the boundary of a compact manifold $X^{n+1}$ endowed with a framing $\tilde{f}$ that restricts on $M$ to $\hat{n} \oplus f$, where $\hat{n}$ is the unit outward-pointing normal field of $M$ in $X$. The inverse $-(M, f)$ of a framed manifold $(M, f)$ is defined by taking $M$ with the "opposite" framing, i.e. with the framing obtained by reversing one of the sections of $f$. Note that this implements the discrete parity symmetry of M-theory on manifolds with vanishing first Spin characteristic class, i.e. on String manifolds and hence framed manifolds. This symmetry is given by an odd number (in this case one) of space and time reflections together with a reflection of the $C$-field $C_{3} \mapsto-C_{3}$. Two framed $n$-manifolds $\left(M_{1}, f_{1}\right),\left(M_{2}, f_{2}\right)$ are framed cobordant if their disjoint union $\left(M_{1}, f_{1}\right) \cap$ $-\left(M_{2}, f_{2}\right)$ is null-cobordant. This is an equivalence relation for framed manifolds, and the set

of equivalence classes of framed $n$-manifolds forms an abelian group $\Omega_{n}^{\mathrm{fr}}$ under disjoint union of manifolds.

Framed cobordism in 9, 10, and 11 dimensions. A closed framed 10-manifold $M^{10}$ represents a class $\left[M^{10}\right] \in \Omega_{10}^{\mathrm{fr}} \cong \pi_{10}^{s}\left(S^{0}\right) \cong \mathbb{Z}_{2} \oplus \mathbb{Z}_{3} \cong \mathbb{Z}_{6}$ via the Pontrjagin-Thom construction. We will also be interested in the 9-dimensional and 11-dimensional cases, for which $\pi_{9}^{s} \cong \mathbb{Z}_{2} \oplus$ $\mathbb{Z}_{2} \oplus \mathbb{Z}_{2}$ and $\pi_{11}^{s} \cong \mathbb{Z}_{7} \oplus \mathbb{Z}_{8} \oplus \mathbb{Z}_{9}$, respectively. The fact that these groups are nonzero implies that there are obstructions to having a framed 9-manifold, 10-manifold, or 11-manifold to be a boundary. However, as in [21] for the Spin case, we will assume that the manifolds that we have are such that there are no such obstructions, i.e., the boundaries are given to us from the start; we will take situations where we have a specific given boundary. By their very construction, non-triviality of the framed bordism groups imply that there are obstructions to having framed manifolds occur as framed boundaries. Even when they do not bound framed manifolds, it might be sufficient that they bound at least a physically admissible manifold, e.g. a Spin manifold.

Lie groups as elements in framed cobordism. If $G$ is a $k$-dimensional compact oriented Lie group then every trivialization of the tangent bundle gives rise to a trivialization of the stable normal bundle and hence to an element of the $k$ th framed cobordism group $\Omega_{k}^{\mathrm{fr}}$. If two choices of linear isomorphisms of the Lie algebra $\mathfrak{g}$ with $\mathbb{R}^{k}$ differ by an element of $\mathrm{GL}(k, \mathbb{R})$ of positive determinant then the corresponding tangential trivializations are homotopic through trivializations and hence determine the same element of $\Omega_{k}^{\mathrm{fr}}$. Therefore, a compact oriented $k$-dimensional Lie group gives rise to a well-defined element $[G] \in \Omega_{k}^{\mathrm{fr}}$.

Adams filtration. A compact Lie group $G$ with its left invariant framing $\mathcal{L}$ defines, via the Pontrjagin-Thom construction, an element $[G, \mathcal{L}]$ in the stable homotopy groups of spheres $\pi_{*}^{s}$. The filtration is a good measure of the complexity of $\pi_{*}^{s}$. For a compact Lie group of rank $r$ the filtration is at least $r$. A result of [38] states that for $G$ a compact Lie group of rank $r$, the 
element $[G, \mathcal{L}]$ in $\pi_{*}^{S}\left(S^{0}\right)$ defined by $G$ in filtration $r$. This filtration is the same as the chromatic level. To detect chromatic phenomena at level 1, that is via K-theory, Lie groups of rank 1 should be used. However, if we want to detect chromatic phenomena at level 2, corresponding to elliptic cohomology (or to Morava K-theory K(2)), we should consider Lie groups of rank 2. Therefore, a priori, the most relevant groups for us will be e.g. $G_{2}, \operatorname{Sp}(2), \mathrm{SO}(5)$, $\mathrm{Spin}(5), \mathrm{SU}(3)$, and their quotients. The element resulting from the Pontrjagin-Thom construction depends only on the orientation of the basis and is denoted by $[G, \alpha, \mathcal{L}]$, where $\alpha$ is the orientation of $G$. Using right translation instead leads to the element $[G, \alpha, \mathcal{R}]$. Sometimes we will leave the orientation out of the notation.

Examples. 1. Spheres. We consider the Lie groups which are spheres, namely $S^{1} \cong \mathrm{SO}(2)$ and $S^{3} \cong \operatorname{Spin}(3)$. The elements $\left[S^{1}, \mathcal{L}\right]$ and $\left[S^{3}, \mathcal{L}\right]$ represent the Hopf maps $\eta \in \pi_{1}^{S}=\mathbb{Z}_{2}$ and $\nu \in \pi_{3}^{S}=\mathbb{Z}_{24}$, respectively.

2. Tori. The three-dimensional torus $T^{3}=S^{1} \times S^{1} \times S^{1}$ represents an element $\eta^{3} \in \pi_{3}^{s}$, where $\eta \in \pi_{1}^{s}$ is the generator represented by $S^{1}$.

3. Central extensions. Let $C$ be a finite central subgroup of $G$ so that there is an extension $C \rightarrow G \rightarrow G / C$. It is natural to ask how the classes $[G, \mathcal{L}]$ and $[G / C, \mathcal{L}]$ might be related. For example, $\mathrm{SO}(3)$ represents $2 \nu$ so that $[\mathrm{SO}(3), \mathcal{L}]=2[\operatorname{Spin}(3), \mathcal{L}]$. In general $[\mathrm{SO}(2 n), \mathcal{L}]=$ $2[\operatorname{Spin}(2 n), \mathcal{L}]$ which is zero for $n \geq 2$ [33]. Other examples of higher dimensions but of rank 2 include $\mathrm{SO}(5), \mathrm{Spin}(5)$ and $\mathrm{Sp}(2)$. In this case $2[\mathrm{SO}(5), \mathcal{L}]=4[\mathrm{Sp}(2), \mathcal{L}]$, as shown in [38]. In terms of generators $[10,38,78,87]$ one has $[\operatorname{Sp}(2), \mathcal{L}]= \pm \beta_{1} \in \pi_{10}^{s} \cong \mathbb{Z}_{6}$. On the other hand, for $\mathrm{SO}(4)$ the class is $[\mathrm{SO}(4), \mathcal{L}]=0$. For the case of the projective group, since $\mathrm{SO}(5) \cong \mathrm{PSp}(2)$ then $2[\operatorname{PSp}(2), \mathcal{L}]=4[\operatorname{Sp}(2), \mathcal{L}]$.

4. Stiefel manifolds. For $1 \leq q \leq n-1$, let $V_{n, q}$ denote the Stiefel manifold of orthogonal $q$-frames in $\mathbb{F}^{n}$, where $\mathbb{F}=\mathbb{R}, \mathbb{C}, \mathbb{H}$. Then, from [50], the element $\left[V_{n, q}, \phi\right]=0$ for a framing $\phi$ in the sense of [45].

5. Flag manifolds. Let $\phi$ be a stable framing of the framed flag manifold $G / T$ and $[G / T, \phi] \in \pi_{*}^{s}$. Then $2[G / T, \phi]=0 \in \pi_{*}^{s}$. This implies that there is a framing $\phi$ of the eightdimensional flag manifold $\operatorname{Sp}(2) / T^{2}$ such that $\left[\operatorname{Sp}(2) / T^{2} ; \phi\right]=\eta \circ \sigma \in \pi_{8}^{s}$, where $\eta \neq 0 \in \pi_{1}^{s}$, as above.

We now highlight a few useful properties of Lie groups elements in framed cobordism.

Properties. 1. Product of groups [10]: For two groups $G$ and $H$ with framings $\mathcal{L}_{G}$ and $\mathcal{L}_{H}$, respectively, the corresponding classes satisfy $\left[G, \mathcal{L}_{G}\right] \times\left[H, \mathcal{L}_{H}\right]=\left[G \times H, \mathcal{L}_{G \times H}\right]$. This implies, for example, that $T^{2}, T^{3}, S^{3} \times S^{3}$, and $S^{3} \times S^{3} \times S^{3}$ with their left-invariant framings give nonzero elements in $\pi_{*}^{s}$.

2. Effect of change of orientation [9]: Changing the orientation results in a possible reversal of sign of the corresponding class $[G, \alpha, \mathcal{R}]=(-1)^{\operatorname{dim} G}[G,-\alpha, \mathcal{L}]=(-1)^{\operatorname{dim} G+1}[G, \alpha, \mathcal{L}]$.

3. Effect of change of representation [51]: For any two real representations $\rho_{1}$ and $\rho_{2}$ of $G$, and for the adjoint representation $\operatorname{Ad}_{G}$, the following holds $\left[G, \operatorname{Ad}_{G}-\rho_{1}+\rho_{2}\right]=(-1)^{\operatorname{dim} G}\left[G, \rho_{1}-\rho_{2}\right]$.

The consequences of the above properties can be summarized in that the only effect at the quantum theory is a possible sign change in the cobordism invariants discussed below. Therefore, if the effective action is already integral then a change in sign would not affect the single-valuedness of the function.

Relation between framed cobordism and Spin cobordism. In positive dimensions, the image of $\Omega_{*}^{\mathrm{fr}} \rightarrow \Omega_{*}^{\text {Spin }}$ is zero unless $*=8 k+1$ or $8 k+2$, where it is $\mathbb{Z}_{2}$ and is detected by the Atiyah-Milnor-Singer $\alpha$-invariant. See [68] for an extensive discussion on the applications to string theory in ten dimensions. Since $\Omega_{11}^{\text {Spin }}=0$, the map $\pi_{11}^{s} \rightarrow \Omega_{11}^{\text {Spin }}$ is trivial and so a framed eleven-dimensional manifold $Y^{11}$ may be viewed as the boundary of a Spin manifold $Z^{12}$ of dimension 12 with the induced Spin structure on $Y^{11}$ being compatible with the framing. 


\subsection{Framed cobordism invariants at chromatic level 1}

We will describe how cobordism invariants of framed manifolds appear in the description of the partition function in M-theory and type II string theory. To that end we first describe these invariants within framed cobordism. In particular, we will describe how the $d$-invariant and the $e$-invariants appear, thus implementing some of the entries appearing in the table in the Introduction.

\subsubsection{The $d$-invariant and Arf invariant in type II string theory}

Here we recall two invariants relevant for the partition function in dimension ten (and to some extent in dimension nine). These two invariants are in fact very closely related.

1. The Arf invariant. Algebraically, Arf invariants are defined for quadratic forms over field of characteristic two and, therefore, occur in various guises. Their most prominent occurrence in topology is that of the Arf-Kervaire invariant of a framed manifold, which is a framed cobordism invariant defined in dimensions $4 k+2, k \geq 0$,

$$
\operatorname{Arf}: \Omega_{4 k+2}^{\mathrm{fr}} \rightarrow \mathbb{Z}_{2}
$$

While this invariant vanishes on closed, framed 10-manifolds, a variant of this invariant is used in the construction of the partition function $[22,52,68]$. The importance of such a variant for type IIB string theory, as well as for the M5-brane, is highlighted in [12, 65]. The Arf-Kevaire invariant is, however, relevant on 6-manifolds. A mathematical discussion on the relation to the M5-brane anomaly (involving manifolds with corners) can be found in [32]. This is further amplified in [69].

2. The $d$-invariant. Using K-theory, Adams defined surjective homomorphisms, the $d$ invariants

$$
d_{\mathbb{R}}: \Omega_{9}^{\mathrm{fr}} \cong \pi_{9} S^{0} \rightarrow \mathbb{Z}_{2}, \quad \Omega_{10}^{\mathrm{fr}} \cong \pi_{10} S^{0} \rightarrow \mathbb{Z}_{2} .
$$

These are given by the mod 2 index of the Dirac operator $[6,8]$. An extensive discussion in the context of M-theory can be found in [68].

Examples. 1. Lie groups. The $d$-invariant $d_{\mathbb{R}}: \pi_{n}^{s} \rightarrow \mathbb{Z}_{2}$, for $n \equiv 1$ or $2 \bmod 8$, vanishes for any non-abelian compact Lie group $G$. In fact, on such a group (e.g. U(3) or $\mathrm{SO}(5)$ ) there is a bi-invariant metric of positive scalar curvature. Hence, by the Lichnerowicz theorem, there are no harmonic spinors on $G$. But from $[7], d_{\mathbb{R}}$ is the real (resp. complex) dimension mod 2 of the space of harmonic spinors on $G$. Thus, $d_{\mathbb{R}}[G]=0$. In fact, if $G$ is a compact Lie group then $d_{\mathbb{R}}([G, \mathcal{L}])=0$ except in low dimensions $[9]$.

2. Finite quotients of Lie groups. Let $G$ be a semisimple Lie group of dimension ten with non-abelian maximal compact subgroup, for example the Lorentz group $\mathrm{SO}(1,4)$. Let $\Gamma$ is a discrete subgroup such that $G / \Gamma$ is compact. Then $d[G / \Gamma]=0[76]$. The same holds for $G$ a simply connected nilpotent Lie group. Now, from Atiyah-Singer index theorem, the $d$-invariant is given by the kernel Ker $D$ of the Dirac operator $D$ on $G / \Gamma$

$$
d[G / \Gamma]=h(G / \Gamma)=\frac{1}{2} \operatorname{dim}(\operatorname{Ker} D) .
$$

It turns out that $h(G / \Gamma)$ is an even integer so that, in the context of $[22,68]$, the partition function is anomaly free.

Note that the result does not apply to general parallelizable manifolds. A counterexample, kindly provided by one of the referees, is the following. By surjectivity of the degree $d_{\mathbb{R}}$, there is a closed framed 9-manifold $M^{9}$ with nontrivial Dirac index modulo two. The circle, equipped with its non-bounding framing, has a nontrivial degree as well. Consequently, the same holds true for the product $M^{9} \times S^{1}$ (with the product framing), which is certainly parallelizable. 


\subsubsection{The $e$-invariant and M-theory}

Using K-theory, Adams defined surjective homomorphisms, the $e$-invariant ${ }^{4}$,

$$
e: \pi_{4 k-1} S^{0} \rightarrow \mathbb{Z}_{d_{k}}, \quad \pi_{8 k} S^{0} \rightarrow \mathbb{Z}_{2},
$$

where $d_{k}$ denotes the denominator of $B_{2 k} / 4 k$, where $B_{i}$ is the Bernoulli number. These numbers are the orders of the corresponding cobordism groups, which are $\Omega_{3}^{\mathrm{fr}} \cong \mathbb{Z}_{24}, \Omega_{7}^{\mathrm{fr}} \cong \mathbb{Z}_{240} \cong$ $\mathbb{Z}_{3} \oplus \mathbb{Z}_{5} \oplus \mathbb{Z}_{16}$ (with generator $S^{7}$ with twisted framing defined by the generator of $\pi_{7}(O) \cong \mathbb{Z}$ ) and $\Omega_{11}^{\mathrm{fr}} \cong \mathbb{Z}_{504} \cong \mathbb{Z}_{7} \oplus \mathbb{Z}_{8} \oplus \mathbb{Z}_{9}$. We are interested in $k=1,2,3$ in the first case and $k=1$ in the second case in (2.5). It is only a low-dimensional 'accident' that the real $e$-invariant can detect all of $\pi_{4 k-1}^{s} S^{0}$ for $1 \leq k \leq 3$. In general, $\mathbb{Z}_{d_{k}}$ occur as direct summands, i.e. the cockerel of the $J$-homomorphism in dimension $4 k-1$ is usually nontrivial.

The $e$-invariant. A $U$-structure is a lift (up to homotopy) of the classifying map of the tangent bundle $T X^{\text {st }}: X \rightarrow$ BO to BU. A ( $U$,fr)-manifold is a compact $U$-manifold $X$ with smooth boundary and a trivialization of $E \cong T X^{\text {st }}$ over the boundary, i.e. a bundle map $\psi$ : $\left.E\right|_{\partial X} \cong \partial X \times \mathbb{C}^{k}$. In particular, $\psi$ provides a framing for $\partial X$. Using relative characteristic classes of the complex vector bundle $E \cong T X^{\text {st }}$, the complex e-invariant of the framed bordism class of $\partial X$ is defined to be

$$
e_{\mathbb{C}}(\partial X) \equiv\langle\operatorname{Td}(E),[X, \partial X]\rangle \bmod \mathbb{Z}
$$

By Atiyah-Patodi-Singer [5] the quantity on the right hand side is $\langle\operatorname{Td}(E),[X, \partial X]\rangle=\int_{X} \operatorname{Td}\left(\nabla^{E}\right)$, where $\nabla^{E}$ is a unitary connection on $E$ which restricts to the canoncial flat connection specified by the trivialization. Similarly, the real $e$-invariant $e_{\mathbb{R}}: \pi_{4 k-1}^{\mathrm{S}} \rightarrow \mathbb{Q} / \mathbb{Z}$ can be defined for Spin manifolds. The two are related by $e_{\mathbb{R}} / \epsilon \equiv e_{\mathbb{C}} \bmod \mathbb{Z}$, where $\epsilon(k)=1$ if $k$ even and $\frac{1}{2}$ otherwise. See $[60,68]$ for applications to M-theory. In that context, since $T Z^{12}$ is trivialized over $Y^{11}$ we can define the relative Pontrjagin classes $p_{i}$ in $H^{4 *}\left(Z^{12}, Y^{11}\right)$ and hence evaluate the $\widehat{A}_{k^{-}}$ polynomial on the fundamental cycle of $Z^{12}$. Then the $e$-invariant of $Y^{11}$ can be defined via the relative $\widehat{A}$-genus as [9]

$$
e\left[Y^{11}\right]=\frac{1}{2} \widehat{A}\left(Z^{12}\right) \quad \bmod \mathbb{Z} .
$$

By the index theorem, this is independent of the choice of the bounding manifold $Z^{12}$. This can be viewed as an analog of the similar observation on the effective action in [22] for the Spin case.

Examples. 1. Spheres. Consider those spheres that are Lie groups. In this case, $e\left[S^{1}\right]= \pm \frac{1}{2}$ and $e\left[S^{3}\right]= \pm \frac{1}{24}$ (see [9]). For applications to the M2-brane see [60].

2. Tori. The 3 -dimensional torus $T^{3}=S^{1} \times S^{1} \times S^{1}$ has $e$-invariant $\frac{1}{2}$. This cannot be directly generalized to higher dimensional tori, due to nil potency, i.e. $\eta^{4}=0$. Therefore, any torus of dimension greater than three represents zero when equipped with the left-invariant framing.

3. Quotients or extensions. For $C$ a central subgroup, knowing the $e$-invariant of the Lie group $G$ allows us to know that of the quotient $G / C$ and vice-versa. For example, the relation $[\mathrm{SO}(3), \mathcal{L}]=2[\operatorname{Spin}(3), \mathcal{L}]=2\left[S^{3}, \mathcal{L}\right]$ gives $e[\mathrm{SO}(3)]= \pm \frac{1}{12}$

4. $\mathbf{S U}(3)$. The group $\mathrm{SU}(3)$ as a framed manifold with a left-invariant framing represents $[\mathrm{SU}(3), \mathcal{L}]=\bar{\nu} \in \pi_{8}^{S}=\mathbb{Z}_{2} \oplus \mathbb{Z}_{2} \cdot \bar{\nu}[80,87]$. Let $\lambda: \mathrm{SU}(3) \rightarrow \mathrm{SU}(3)$ denote the identity map regarded as the fundamental representation of $\mathrm{SU}(3)$ on $\mathbb{C}^{3}$. Then the $e$-invariant of $[\mathrm{SU}(3), \lambda]$ is nonzero [87].

\footnotetext{
${ }^{4}$ Note that there is a third variant of the Adams $e$-invariant, viz. $e: \operatorname{ker}\left(d_{\mathbb{R}}: \pi_{8 k+1}^{s} S^{0} \rightarrow \mathbb{Z}_{2}\right) \rightarrow \mathbb{Z}_{2}$, detecting the image of the $J$-homomorphism in these dimensions.
} 
5. U(3). Let $\sigma$ be the generator of $\pi_{2}^{s}\left(B S^{1}\right)$ given by the Hopf bundle. Then $t(\sigma)=\left[S^{3}, \mathcal{L}\right]$ generates $\pi_{3}^{s}\left(S^{0}\right)$, so that $t(3 \sigma)=\nu$. From $\eta \bar{\nu}=\nu^{3}$, this gives $[\mathrm{U}(3), \mathcal{L}]=\eta[\mathrm{SU}(3), \mathcal{L}]=\nu^{3}$.

Integrality and corners. The Todd genus of a $(U, \mathrm{fr})$-manifold is integral if and only if the complex $e$-invariant $e_{\mathbb{C}}$ of its boundary is integral or, equivalently, if and only if its boundary is the corner $\partial_{0} \partial_{1} X$ of a $(U, \mathrm{fr})^{2}$-manifold $X$. In the context of M-theory, this says that the index - in the form of the Todd genus - of $Z^{12}$ is integral if and only if $Y^{11}=\partial_{0} \partial_{1} W^{13}$ of a $(U, \mathrm{fr})^{2}$-manifold $W^{13}$. This views $Y^{11}$ itself as a corner, in contrast to viewing its boundary as a corner, as we do for most of this article. The structure of the topological terms in M-theory indeed do not suggest a lifting to thirteen dimensions.

\subsection{Change of framing}

Like other geometric structures on manifolds (e.g. Spin structure), a framed manifold might admit different framings. In this section we will study the possible physical effect of the change of framing. The analog for Spin and $\mathrm{Spin}^{c}$ structures is studied in [68], and that of String structures in [60].

A framing on a manifold $M$ corresponds to a lift in the diagram

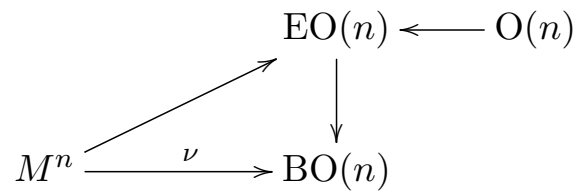

The different choices of framing correspond to maps from $M^{n}$ to the fiber $\mathrm{O}(n)$ of the principal classifying bundle. Given a framed manifold $M^{n}$ and a map $\mathcal{F}: M^{n} \rightarrow \mathrm{O}(n)$ we may use $\mathcal{F}$ to change the framing. Conversely, given two framings $\phi_{1}$ and $\phi_{2}$ of $M$, they differ by a map $\phi_{2} / \phi_{1}: M^{n} \rightarrow \mathrm{O}(n)$. The change of framing in the case of two and ten dimensions can be viewed from the point of view of the Atiyah $\alpha$-invariant $[60,68]$ which is the refinement of the $\bmod 2$ index of the Dirac operator from $\mathbb{Z}_{2}$ to $\mathrm{KO}_{m} \cong \mathbb{Z}_{2}$ for $m=2,10$.

Twisted framing. We start with the case of a Lie group, which is always oriented as a manifold. Given a map $\varphi: G \rightarrow \mathrm{SO}(n)$, there is an automorphism of the trivial bundle $G \times \mathbb{R}^{d}$ given by $(g, \omega) \mapsto\left(g, \varphi g^{-1}(w)\right)$. Then the twisted framing (here for right) $\mathcal{R}^{\varphi}$ of $\mathcal{R}$ by $\varphi$ is defined as the direct sum of $\mathcal{R}$ with this automorphism. The determination of $\left[G, \mathcal{R}^{\varphi}\right]$ depends essentially on the element of the reduced group $\widetilde{\mathrm{KO}}^{-1}\left(G^{+}\right)$represented by $\varphi$, where $G^{+}$is $G$ with a point adjoined. Given an element $\alpha$ in $\widetilde{\mathrm{KO}}^{-1}(G)$ we may twist a given framing $\phi$ of $G$ to obtain a new element $\left[G, \phi^{a}\right]$. Let $M^{n}$ be a framed manifold embedded in $\mathbb{R}^{n+k}$ and $\phi$ a framing of its normal bundle $N M$. For $\alpha \in \widetilde{\mathrm{KO}}^{-1}(M)$ the twisted framing $\phi^{\alpha}$ is constructed as the composition of $\phi$ and the automorphism $\tilde{\alpha}$ of the trivial bundle $M \times \mathbb{R}^{k}$ determined by $\alpha$.

Example. Change of framing on sphere bundles over spheres. We consider the example we discussed earlier in Sections 2.1 and 2.2. The framing $\phi$ of $S(E)$ is called the induced framing. All other framings of $S(E)$ may be obtained from $\phi$ by twisting with elements of $\widetilde{\mathrm{KO}}^{-1}(S(E))$. Suppose that $[E]=0 \in \widetilde{\mathrm{KO}}\left(S^{n}\right)$. Then $S(E)$ is a $\pi$-manifold and hence there are elements $[S(E) ; \phi] \in \pi_{n+m-1}^{s}$ corresponding to the different framings $\phi$ of $S(E)$.

Change of framing and the $\boldsymbol{d}$-invariant. The change of framing will have an effect on the $d$-invariant. We consider the case of a Lie group, i.e. a WZW model context. For an element $\alpha$, giving rise to a left framing $\mathcal{L}^{\alpha}$, the $d$-invariant will get modified by $J(\alpha)$ as

$$
d_{\mathbb{R}}\left(\left[G, \mathcal{L}^{\alpha}\right]\right)=d_{\mathbb{R}}(J(\alpha)) \circ d_{\mathbb{R}}([G, \mathcal{L}]) .
$$

In order to guarantee the absence of mod 2 anomalies in the partition function, we would like the transformed $d$-invariant to be even. If $d_{\mathbb{R}}([G, \mathcal{L}])$ started out as being already even then 
there are no conditions needed. However, if $d_{\mathbb{R}}([G, \mathcal{L}])$ were odd then there are no potential anomalies if $d_{\mathbb{R}}(J(\alpha))$ is also even. If this occurs, then the change of framing could be viewed as a way of curing an anomaly.

Note that, due to the index interpretation, questions about the degree can be rephrased in terms of Spin geometry (see [68] for extensive discussion in the context of M-theory). In particular, for WZW models on compact non-abelian groups, the scalar curvature argument ensures triviality of the degree (independent of the framing/Spin structure). On the abelian groups $S^{1}$ and $S^{1} \times S^{1}$, however, it is easy to write down reframings which change the Spin structures and the degree. Unfortunately, these targets do not give rise to 'honest' WZW models.

Change of framing and the $e$-invariant. Let $s^{*}: H^{*}(\mathrm{BSO} ; \mathbb{Q}) \rightarrow H^{*}(\mathrm{SO} ; \mathbb{Q})$ be the cohomology suspension and set $u_{n}=s^{*} p_{n} \in H^{4 n-1}(\mathrm{SO} ; \mathbb{Q})$ where $p_{n} \in H^{4 n}(\mathrm{BSO} ; \mathbb{Q})$ is the $n$th universal Pontrjagin class. The cohomology suspension kills decomposable elements ${ }^{5}$ so that

$$
s^{*} \widehat{A}_{n}(p)=-\frac{B_{n}}{4 n} \frac{s^{*} p_{n}}{(2 n-1) !}=-\frac{B_{n}}{4 n} \frac{u_{n}}{(2 n-1) !},
$$

where $B_{n}$ is the $n$th Bernoulli number. Suppose that $E \rightarrow S^{n}$ is stably trivial $m$-plane bundle, with $\phi$ a stable trivialization of $E$, and $\alpha \in \widetilde{\mathrm{KO}}^{-1}(S(E))$. Then putting $4 k=n+m$, the $e$-invariant with new element is [78]

$$
e\left[S(E) ; \phi_{\alpha}\right]=-\frac{a_{k} B_{k}}{4 k(2 k-1) !}\left\langle\alpha^{*} u_{k},[S(E)]\right\rangle .
$$

Since Lie groups are admissible manifolds which, furthermore, do not lead to anomalies in the partition function, we should have that $\alpha^{*} u_{3}$ is an even multiple of $u_{3}$ for an 11-dimensional bundle with a spherical base.

Examples. 1. Let $G$ be a compact connected Lie group of dimension 3 and $\alpha \in \widetilde{\mathrm{KO}}^{-1}(G)$ be an element with second Stiefel-Whitney class $w_{2}(\alpha)$ zero. If $p_{1}(\alpha)$ is the first Pontragin class of $\alpha$ in $H^{3}(G ; \mathbb{Z})$ (i.e. via transgression to the Chern-Simons form) then the $e$-invariant of the variation is given by [38]

$$
e_{\mathbb{R}}\left(\left[G, \mathcal{L}^{\alpha}\right]-[G, \mathcal{L}]\right)=-\left\langle\frac{1}{4} B_{2} \cdot p_{1}(\alpha),[G]\right\rangle_{H} \bmod 2 \mathbb{Z} .
$$

From the results in [68], we require the cohomological pairing on the right hand side of (2.6) to be an integer. This places an obvious congruence condition on the Pontrjagin class $p_{1}(\alpha)$. The groups we have in mind are $\mathrm{SU}(2) \cong \operatorname{Spin}(3) \cong \mathrm{Sp}(1)$ and $\mathrm{SO}(3)$.

2. If $G$ is a compact connected Lie group of dimension $m=8$ or 9 , and $\lambda \in \widetilde{\mathrm{KO}}^{-1}(G)$ satisfies $w_{2}(\lambda)=0$ then $[87]$

$$
e_{\mathbb{R}}\left(\left[G, \mathcal{L}^{\lambda}\right]-[G, \mathcal{L}]\right)=\left\langle\rho^{3}\left(E_{\lambda}\right)-1,[G]\right\rangle_{\mathrm{KO}} \in \mathrm{KO}_{m+1}(*) \cong \mathbb{Z}_{2} .
$$

Here $\rho^{3}$ is the cannibalistic characteristic class of Adams and Bott associated with the Thom isomorphism $\rho^{3}(E)=\phi_{E}^{-1} \circ \psi^{3} \circ \phi_{E}(1)$. The groups we have in mind here are $\mathrm{SU}(3)$ in dimension 8 and $\mathrm{U}(3)$ in dimension 9. The Adams operation $\psi^{i}$, which is an automorphism of K-theory, is given a physical interpretation in the context of M-theory in [68]. As in the previous case, the results of [68] require that the KO-theoretic pairing on the right hand side of (2.7) to be zero in $\mathbb{Z}_{2}$.

The consequence of the above two examples is that the difference of the $e$-invariant should be an even integer in order for the partition function to be anomaly-free.

\footnotetext{
${ }^{5}$ This is a process used in [64].
} 


\section{Topological and modular aspects of M-theory with corners}

\subsection{Building the general setting in M-theory}

We will show in this section how the formulation in terms of corners is the natural setting for topological considerations of M-theory. This complements the analytical point of view in [63].

Consider M-theory on an 11-dimensional manifold $Y^{11}$ with boundary $M^{10}=\partial Y^{11}$. This is considered in [21], where a model of the $C$-field which is valid in that case is proposed, with the phase given by

$$
\Phi\left(C, Y^{11}\right)=\exp \left[i \pi \xi\left(D_{A}\right)+\frac{i \pi}{2} \xi\left(D_{\mathrm{RS}}\right)+2 \pi i I_{\text {local }}\right]
$$

where $\xi\left(D_{A}\right)$ is a section of a $\mathrm{U}(1)$ bundle with connection over the space of $C$-fields on the boundary $M^{10}$, with $D_{A}$ an $E_{8}$ Dirac operator and $D_{\mathrm{RS}}$ the Rarita-Schwinger operator, and $\xi$ denotes the reduced eta-invariant $\xi(D)=\frac{1}{2}(\eta(D)+h(D))$. The model is also extended to the case when the 11-manifold is of the form $Y^{11}=X^{10} \times S^{1}$ with the type IIA 10-manifold $X^{10}$ itself having a boundary $N^{9}=\partial X^{10}$, so that $M^{10}=N^{9} \times S^{1}$.

The authors of [21] also consider the reduction of the phase from $Z^{12}$ to $M^{10}$ in the product case $^{6}$. With the ansatz for the field strength $G_{Z}=G_{M}+d t_{1} \wedge \omega_{1}+d t_{2} \wedge \omega_{2}$, the phase reduced to $M^{10}$ takes the form $\exp \left[-2 \pi i \frac{1}{2} \int_{M^{10}} G \wedge \omega_{1} \wedge \omega_{2}\right]$. We will propose a generalization of this, in some sense, to manifolds with corners. The authors consider conditions on torsion cohomology classes; we will in addition be interested in doing global analysis. As the structure above suggests, we will highlight the case when the corner $M^{10}$ is the total space of a circle bundle $\pi$. The pairing $H^{7}\left(N^{9} ; \mathbb{Z}\right) \times H^{2}\left(N^{9} ; U(1)\right) \rightarrow U(1)$ suggests that the structure of the base of that circle bundle $\pi$ has a 7 -dimensional factor. Indeed, the most interesting case will be when $M^{10}=S^{3} \times S^{7}$, corresponding to a base $N^{9}=S^{7} \times S^{2}$ or $N^{9}=\mathbb{C} P^{3} \times S^{3}$.

We generalize the above setting of product structure to more general bundle structure captured by this schematic diagram

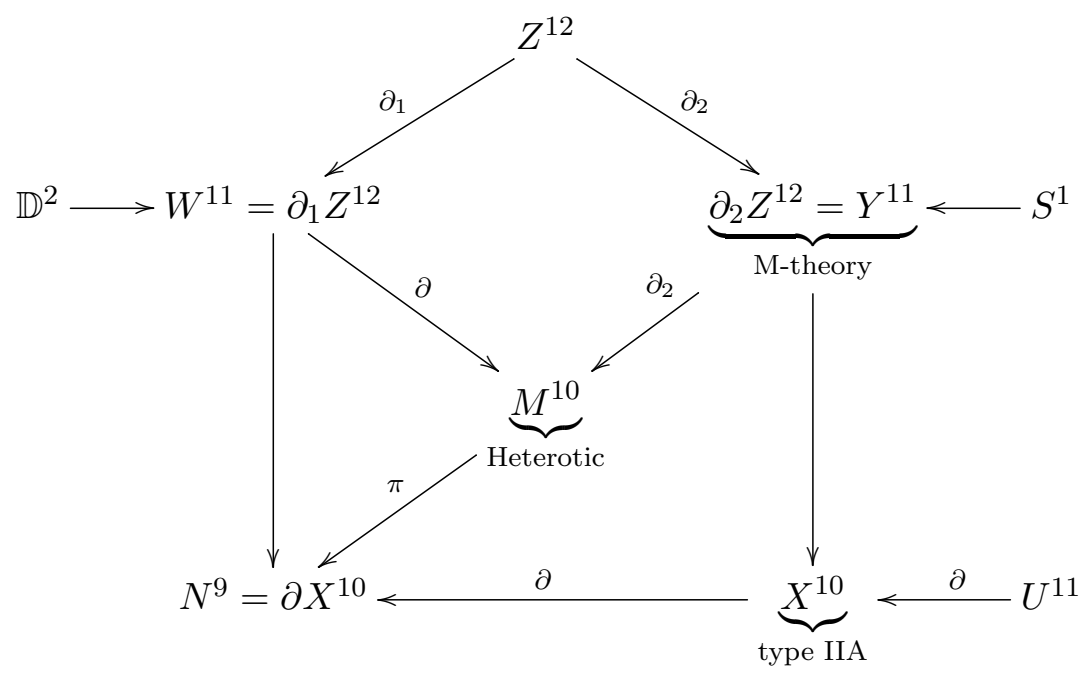

The extension of type IIA to $U^{11}$ is discussed in $[12,59]$. General boundary conditions on the $C$-field (and its dual) are studied in [67].

The Rarita-Schwinger field and supersymmetry. We highlight some effect on the spinor fields and, in particular, as far as stable trivialization is involved. The Rarita-Schwinger operator over a manifold $M$ is defined as the Dirac operator twisted by the virtual bundle

\footnotetext{
${ }^{6}$ They consider $Z^{12}=M^{10} \times \Delta^{2}$, where $\Delta^{2}$ is a 2-simplex $\left\{\left(t_{1}, t_{2}\right): 0 \leq t_{1} \leq t_{2} \leq 1\right\}$ with identification $t_{i} \sim t_{i}+1$.
} 
$R S=T M \ominus m \mathcal{O}$, for some multiple of the trivial line bundle $\mathcal{O}$. For example, $m=4,3,2$ in twelve, eleven, and ten dimensions, respectively. Had the twisting bundle $R S$ not been a virtual bundle, it would have been stably trivial if $R S \oplus n \mathcal{O}$ is trivial. This then would imply that $T M$ is trivial, and hence that $M$ is parallelizable (see Section 2.1). However, $R S$ is only a virtual bundle and, as such, triviality only makes sense in $\widetilde{\mathrm{KO}}(M)$ and, in particular, such a triviality cannot be used to deduce strict parallelizability of $M$. Of course, if $T M$ is trivial then $R S$ would be. In this case, the characteristic classes of the tangent (and Spin) bundle will be trivial. Triviality of the tangent bundle implies the triviality of the Spin bundle. Parallelizability implies the maximum number of linearly independent sections. Thus, for the Spin bundle this ensures the maximum number of spinors. This is desirable for compactification to low dimensions.

\subsection{Relation to type IIA: Disk bundles and eta-forms}

In this section we will consider the effective action and partition function of type IIA string theory in the context of corners. We will identify the contribution to the phase. This will allow us to provide physical interpretation of higher eta-forms, extending the discussion in [48, 59].

Let $Z$ be the total space of a disk bundle over a $\pi$-manifold $X$ associated with a vector bundle $E$. For the purpose of relating M-theory to type IIA string theory, $E$ is usually a hermitian line bundle. The Kervaire semi-characteristic is zero, so that if the circle bundle $Y^{11}$ is stably parallelizable then it is also parallelizable.

$Z^{\mathbf{1 2}}$ as a $\langle\mathbf{2}\rangle_{f}$-manifold. We consider $Z^{12}$ as a fiber bundle $\pi: Z^{12} \rightarrow X^{10}$, where both the disk fiber $\mathbb{D}^{2}$ and the type IIA base $X^{10}$ are compact $\langle 1\rangle$-manifolds, i.e. manifolds with boundary, and the faces are given by the fiber bundles (see diagram (3.1))

$$
\begin{array}{ll}
\partial_{1} Z^{12}=W^{11}: & \mathbb{D}^{2} \rightarrow W^{11} \rightarrow \partial X^{10}=N^{9}, \\
\partial_{2} Z^{12}=Y^{11}: & S^{1}=\partial \mathbb{D}^{2} \rightarrow Y^{11} \rightarrow X^{10} .
\end{array}
$$

Consider metrics $g^{T X}$ and $g^{T D}$ and connections $\nabla^{T X}$ and $\nabla^{T D}$ on $T X^{10}$ and $T \mathbb{D}^{2}$, respectively. Corresponding to the splitting $T Z^{12} \cong T \mathbb{D}^{2} \oplus \pi^{*} T X^{10}$, we have a connection $\nabla^{\oplus}=\nabla^{T D} \oplus$ $\pi^{*} \nabla^{T X}$. Consider a Dirac family $D_{E}$ on $Z^{12}$ parametrized by points on the base, as in $[48,59$, $64,67,63]$, assuming a metric of product type near the boundary. Furthermore, we assume that the kernel of $D_{E}$ induced on the fiberwise boundary (3.2) is of constant rank, so that the index bundle with respect to the Atiyah-Patodi-Singer boundary problem is well-defined, with $D_{E}^{\partial}$ the corresponding twisted Dirac operator on the boundary. We also consider the line bundle $L$ with connection $\nabla^{L}$ corresponding to the principal circle bundle (see [68]).

Contribution to the phase from the boundary of type IIA. The Bismut-Cheeger [13] and Melrose-Piazza [49] formulation of the index implies that a representative in cohomology of the Chern character of the index bundle is given by the following differential form on $X^{10}$ :

$$
\int_{\mathbb{D}^{2}}\left\{\widehat{A}\left(\nabla^{T D}\right) \operatorname{ch}\left(\nabla^{L^{1 / 2}}\right) \operatorname{ch}\left(\nabla^{V}\right)\right\}-\widehat{\eta}\left(D_{V}^{\partial}\right)-\frac{1}{2} \operatorname{ch}\left(\nabla^{\operatorname{ker} D_{V}^{\partial}}\right) .
$$

The Index class can be encoded in a virtual vector bundle $\xi$ with unitary connections $\nabla^{\xi}$, such that $[\xi]=\left[\xi_{1} \ominus \xi_{2}\right]=\left[\right.$ Ind], so that in de Rham cohomology $(\operatorname{ch}(\operatorname{Ind}))_{\mathrm{dR}}=\operatorname{ch}\left(\nabla^{\xi}\right)+d \omega$, for some $\omega \in \Omega^{\text {odd }}\left(X^{10}\right)$. We now introduce the $E_{8}$ bundle $V$ with connection $\nabla^{V}$ on $Z^{12}$ and its restriction to the boundary, with the same notation, and assuming a pull-back structure, i.e. with the bundle $E$ pulled back fro the base. This is a reasonable assumption from the physical point of view, as in $[22,48,59]$. Furthermore, we assume that the Dirac family on the (fiberwise) boundary should be invertible ${ }^{7}$. Then, with a product connection $\nabla^{\oplus}$, via [83]

$$
\int_{Z^{12}} \widehat{A}\left(\nabla^{\oplus}\right) \operatorname{ch}\left(\pi^{*} \nabla^{V}\right)=\int_{Z^{12}} \widehat{A}\left(\pi^{*} \nabla^{T X}\right) \widehat{A}\left(\nabla^{T D}\right) \operatorname{ch}\left(\pi^{*} \nabla^{V}\right)
$$

\footnotetext{
${ }^{7}$ Alternatively, one could absorb additional corrections by redefining the $\widehat{\eta}$-forms.
} 


$$
\begin{aligned}
& =\int_{X^{10}}\left\{\widehat{A}\left(\nabla^{T X}\right) \operatorname{ch}\left(\nabla^{V}\right)\left(\operatorname{ch}\left(\nabla^{\xi}\right)+d \omega+\widehat{\eta}\right)\right\} \\
& =\operatorname{Ind}\left(D_{\xi \otimes V}\right)+\int_{X^{10}} \widehat{A}\left(\nabla^{T X}\right) \operatorname{ch}\left(\nabla^{V}\right) \widehat{\eta}+\left\{\xi\left(D_{\xi \otimes V}^{\partial X}\right)+\int_{\partial X^{10}} \omega \widehat{A}\left(\nabla^{T X}\right) \operatorname{ch}\left(\nabla^{V}\right)\right\} .
\end{aligned}
$$

The phase of the partition function is Phase $=\operatorname{Ind}\left(D_{\xi \otimes V}\right)$. The last term in (3.3) is the contribution from the boundary in type IIA.

Interpretation of the eta-forms. The basic part of expression (3.3) gives

$$
\int_{\mathbb{D}^{2}} \widehat{A}\left(\nabla^{T D}\right)=(\operatorname{ch}(\text { Ind }))_{\mathrm{dR}}+\widehat{\eta} .
$$

For the three relevant nontrivial degrees we have

$$
\begin{aligned}
& \int_{\mathbb{D}^{2}} \widehat{A}_{4}\left(\nabla^{T D}\right)=\left(\operatorname{ch}_{1}(\text { Ind })\right)_{\mathrm{dR}}+a B_{2}, \\
& \int_{\mathbb{D}^{2}} \widehat{A}_{8}\left(\nabla^{T D}\right)=\left(\operatorname{ch}_{3}(\text { Ind })\right)_{\mathrm{dR}}+b B_{2}^{3}, \\
& \int_{\mathbb{D}^{2}} \widehat{A}_{12}\left(\nabla^{T D}\right)=\left(\operatorname{ch}_{5}(\text { Ind })\right)_{\mathrm{dR}}+c B_{2}^{5},
\end{aligned}
$$

where we identify the components of the eta-form as powers of the $B$-field. The numerical coefficients $a, b, c$ can be read off for a certain class of situations; for instance, with a suitable identification of the $B$ and $F$ fields and using expressions (3.4) below. This generalizes to higher degrees the interpretation in $[48,59]$ of the eta-form in degree two as essentially the $B$-field.

We can approach this from an another angle which makes it a bit more physically explicit. Consider type IIA string theory on $U^{8} \times T^{2}$ with metric $d s_{X}^{2}=d s_{T^{2}}^{2}+t^{2} d s_{U}^{2}$. When the $E_{8}$ bundle is trivial and with the Ramond-Ramond fields $F_{0}=0=F_{4}$ then the phase in this case, via [52], is

$$
\Phi_{1}=\int_{X^{10}}\left[-\frac{1}{15}\left(F_{2}\right)^{5}+\frac{1}{3}\left(F_{2}\right)^{3} \cdot \frac{1}{24} p_{1}-\frac{1}{2} F_{2} \cdot \widehat{A}_{8}\right] .
$$

Comparing with the second term in (3.3), we have the following interpretation of the eta-forms

$$
\widehat{\eta}_{2}=-\frac{1}{2} F_{2}, \quad \widehat{\eta}_{6}=-\frac{1}{3}\left(F_{2}\right)^{3}, \quad \widehat{\eta}_{10}=-\frac{1}{15}\left(F_{2}\right)^{5} .
$$

This is another interpretation of eta-forms in terms of the Ramond-Ramond 2-form rather than in terms of the $B$-field. However, there is no conflict as the two fields can be identified for some topological purposes; see [66, 68].

\subsection{The heterotic theory as a corner}

In this section we will provide another argument for why the corner setting is natural to consider for heterotic string theory. This complements the discussion above in Section 3.1 as well as the analytic arguments in [63]. In particular, the structure of the heterotic anomaly points in a natural way to a corner formulation. The heterotic theory, like other flavors of string theory, will mostly arise on manifolds which are decomposable as products or as fiber bundles, as in [67].

The heterotic corner as a framed cobordism class. We will consider the heterotic corner as a framed corner. The invariant we will associate to this is a framed cobordism invariant, and hence depends only on the framed cobordism class. Using the results of [19], the data that refines the corner $M^{10}$ into a representative of a framed cobordism class

$$
\left[M^{10}\right] \in \Omega_{12}^{(U, \text { fr })^{2}}
$$

and which interestingly matches the physical setting (see diagram (3.1)), is the following 
1. A decomposition $T M^{10} \cong T^{0} M^{10} \oplus T^{1} M^{10}$ of framed bundles. This implements a factorization, as e.g. dictated by anomaly cancellation (see below).

2. Compact 11-dimensional manifolds $W^{11}, Y^{11}$ with boundary $\partial W^{11} \cong-\partial Y^{11}$. The manifold $Y^{11}$ where M-theory resides, while $W^{11}$ is 'physically hidden'.

3. Decompositions $T W^{11} \cong T^{0} W^{11} \oplus T^{1} W^{11}$ with complex structure on the first factor and framing on the second factor; and $T Y^{11} \cong T^{0} Y^{11} \oplus T^{1} Y^{11}$ with framing on the first factor and complex structure on the second factor. For instance, in the product $Y^{11}=X^{3} \times W^{8}$, we take $X^{3}$ to be framed and $W^{8}$ to be complex (e.g. a Calabi-Yau manifold).

4. The inclusions $M^{10} \hookrightarrow W^{11}, M^{10} \hookrightarrow Y^{11}$ identify

$$
\begin{aligned}
& \left.\left(T^{1} W^{11}\right)\right|_{M^{10}} \cong T^{1} M^{10},\left.\quad\left(T^{0} W^{11}\right)\right|_{M^{10}} \cong T^{0} M^{10}, \quad \text { as framed bundles, } \\
& \left.\left(T^{0} W^{11}\right)\right|_{M^{10}} \cong T^{0} M^{10},\left.\quad\left(T^{1} W^{11}\right)\right|_{M^{10}} \cong T^{1} M^{10}, \quad \text { as complex bundles. }
\end{aligned}
$$

5. A manifold with corners $Z^{12}$ such that $\partial_{0} Z^{12} \cong W^{11}$ and $\partial_{1} Z^{12} \cong Y^{11}$. This is the lift in diagram (3.1).

6. A decomposition $T Z^{12} \cong T^{0} Z^{12} \oplus T^{1} Z^{12}$ of complex vector bundles such that

7. The inclusions $W^{11} \hookrightarrow Z^{12}$ and $Y^{11} \hookrightarrow Z^{12}$ identify

$$
\begin{aligned}
& \left.T^{0} Z^{12}\right|_{W^{11}} \cong T^{0} W^{11},\left.\quad T^{1} Z^{12}\right|_{W^{11}} \cong T^{1} W^{11}, \\
& \left.T^{0} Z^{12}\right|_{Y^{11}} \cong T^{0} Y^{11},\left.\quad T^{1} Z^{12}\right|_{Y^{11}} \cong T^{1} Y^{11},
\end{aligned}
$$

as complex bundles. Again, the identifications (3.5), (3.6) are to be understood as identifications of stable bundles.

The 11-dimensional manifold $W^{11}$ is to satisfy the above conditions so that diagram (3.1) is properly implemented.

\subsubsection{Corners as the natural setting for heterotic anomalies}

Consider the Green-Schwarz anomaly in the heterotic (and type I) theory. The general formulation of anomalies requires considering a degree twelve polynomial whose integration over the 10-dimensional manifold is the curvature of the anomaly line bundle. Consider heterotic string theory on a Spin manifold $\left(M^{10}, g\right)$ with Spin bundle $S M$ and with a vector bundle $E$ of structure group $E_{8} \times E_{8}$ or $\operatorname{Spin}(32) / \mathbb{Z}_{2}$, which captures the dynamics of the Yang-Mills fields via a connection $A$ on $E$. Then the $H$-field and its dual satisfy [27]

$$
\begin{aligned}
& \frac{1}{2 \pi} d\left(H_{3} u^{-2}+H_{7} u^{-4}\right)=\left[\operatorname{ch}_{2}(A)-p_{1}(g)\right] u^{-2} \\
& \quad+\left[\operatorname{ch}_{4}(A)-\frac{1}{48} p_{1}(g) \operatorname{ch}_{2}(A)+\frac{1}{64} p_{1}(g)^{2}-\frac{1}{48} p_{2}(g)\right] u^{-4},
\end{aligned}
$$

where $u$ is the Bott generator. The anomaly polynomial is given by

$$
I_{12}=\int_{Z^{12}} X_{4} \wedge X_{8}
$$

with $H_{3}$ and $H_{7}$ providing the trivializations of the forms $X_{4}$ and $X_{8}$, respectively, as given in (3.7). The usual connection to ten dimensions is that one has to introduce a local term in the action functional of the form $\int_{M^{10}} B_{2} \wedge X_{8}$. Our interpretation of this is that it is implicit that we schematically have the following relations

$$
X_{4}=d H_{3}, \quad H_{3}=d B_{2},
$$


which hints at a direct relation between $X_{4}$ and $B_{2}$ had one been able to make sense of a boundary of a boundary. With the lack of this, the formulation in terms of corners seems to be an alternative. Note that one still cannot directly take a boundary of a boundary, neither in the homological or the cohomological setting. A proper interpretation at each step is given towards the end of Section 2.5. A similar argument holds for the dual field $H_{7}$ with the polynomial $X_{8}$. Therefore, the structure of the anomaly and the process of anomaly cancellation have a natural home in the setting of manifolds with corners. Such a formulation also holds in other situations where topological anomaly cancellation occurs. While we do not explicitly spell them out, it is obvious how our discussion could be adapted.

Note that the anomaly polynomial can be given in terms of the elliptic genus as [44]

$$
I_{12}=\left.\phi_{\text {ell }}\right|_{12 \text {-form coeff. of } q^{0}} \text {. }
$$

Then a natural question is what this corresponds to in ten dimensions. Our formulation in terms of corners can also be viewed as providing an answer to this question. Note that the two terms in the integrand in (3.8) are interpreted in terms of twisted string structure and twisted Fivebrane structures, respectively [71, 72].

\subsubsection{The one-loop term in the presence of a corner}

The topological study of M-theory relies on the existence of the one-loop polynomial $I_{8}$ [24], which is a degree eight polynomial in the Pontrjagin classes of eleven-dimensional spacetime $Y^{11}$. The term in the action is of the form $\int_{Y^{11}} C_{3} \wedge I_{8}$. It is natural to ask how this term behaves under dimensional reduction to ten or lower dimensions, or upon dimensional lifting to twelve and higher dimensions. An example of the former is in type IIA string theory, where the term takes the form $\int_{X^{10}} B_{2} \wedge I_{8}$. An example of the latter situation is the lift to the 12-dimensional bounding theory on $Z^{12}$, where the term takes the form $\int_{Z^{12}} G_{4} \wedge I_{8}$. Extensions beyond twelve dimensions are considered in [57]. What we would like to analyze is how this term behaves upon reduction from $Z^{12}$ to its 10-dimensional corner $M^{10}$.

The one-loop term lifted to twelve dimensions takes the form

$$
G_{4} \wedge I_{8}=\left(\frac{1}{2} \lambda-a\right) \wedge\left(\frac{1}{48}\left(p_{2}-\lambda^{2}\right)\right), \quad \lambda=\frac{1}{2} p_{1} .
$$

We will concentrate at the prime $p=3$, i.e. work 3 -adically, so that the obstruction to the Fivebrane structure $\frac{1}{6} p_{2}$, in the sense of [71], as well as the term $\frac{1}{48} p_{2}$ in expression (3.9) both can be effectively viewed as the fractional class $\frac{1}{3} p_{2}$. We will concentrate on the $\lambda \wedge p_{2}$-term in (3.9). Passing to the complex case, taking into account the fact that we are taking $Z^{12}$ to be a complex manifold, this part of the action can be written in terms of the Chern classes of $Z^{12}$. Indeed, if $E$ is a general complex vector bundle then for the underlying real bundle $E_{\mathbb{R}}$ one has

$$
p_{1}\left(E_{\mathbb{R}}\right) \equiv-2 c_{2}(E) \quad \bmod c_{1}(E) \quad \text { and } \quad\left(p_{2}-\lambda^{2}\right)\left(E_{\mathbb{R}}\right) \equiv 2 c_{4}(E) \quad \bmod c_{1}(E) .
$$

So if we impose the condition that $E$ is an SU-bundle so that $c_{1}(E)=0$ (this is satisfied in the physically favorable setting of the tangent bundle of Calabi-Yau manifolds), then we get for the action

$$
\int_{Z^{12}} c_{2} \cdot \frac{1}{3} c_{4}
$$

This Chern number can be viewed as an analog of the term $\int_{X^{10}} \frac{1}{2} c_{2} \cdot c_{3}$ that appears in the calculation of the partition function in type IIA via K-theory and $E_{8}$ gauge theory [22]. The expression (3.10) is a 12-dimensional analog with the prime 3 taking the place of the prime 2 . 
Now we reduce expression (3.10) to the corner $M^{10}$. The general form of the term at the level of differential forms is

$$
\int_{M^{10}} \frac{1}{3} C S_{3} \wedge C S_{7}
$$

a product of a Chern-Simons 3-form and a Chern-Simons 7 -form, both of gravitational type. We would like to see how this fits into the description of the corner via the $f$-invariant (see Section 3.5). The construction of the $f$-invariant requires a presentation of the framed tenmanifold $M^{10}$ as a corner of codimension two of an almost complex twelve-manifold $Z^{12}$ with suitable splitting of the stable tangent bundle. We illustrate this with the main physical example, which turns out to also be the main example in the mathematical construction of the $f$-invariant in [42]. The group $\operatorname{Sp}(2)$ admits different framings. Equipped with its left-invariant one, $\operatorname{Sp}(2)$ represents a generator of $\pi_{10}^{s} S_{(3)}^{0}$, i.e. the element $\beta$ at the prime $3[38,42]$. Note that there is also a bounding one, as stated previously. This example, which is a 3 -sphere bundle over the 7-sphere, is relevant in string theory in relation to nonrepresentable cycles [25].

Example: $M^{\mathbf{1 0}}=\mathbf{S p}(\mathbf{2})$. This example is described from the point of view of K-theory at the prime 3 in [25]. We will derive conditions on the (lifted) one-loop term arising from a condition for $\operatorname{Sp}(2)$ to be the corner of the 12 -manifold $Z^{12}$. Let $\left[Z^{12}, \partial Z^{12}\right]$ be the relative fundamental class. Pairing cohomology classes with this homology class amounts to detecting those classes which are nontrivial on $Z^{12}$ but become trivial on the boundary $Y^{11}$. Such a situation is described in detail in [64]. Now let us consider the condition for the corner to be $\operatorname{Sp}(2)$. To that end, we will take $Z^{12}$ as a (U,fr) ${ }^{2}$-manifold with a splitting of the stable tangent bundle $T^{1} Z^{12} \oplus T^{1} Z^{12}$, and consider the corresponding Chern classes $\left\{c_{1}, \ldots, c_{6}\right\}$ and $\left\{\tilde{c}_{1}, \ldots, \tilde{c}_{6}\right\}$ of the two subbundles $T^{0} Z^{12}$ and $T^{1} Z^{12}$, respectively. The condition derived in [42] is given by the $f$-invariant and depends on all possible Chern numbers of total degree 6 ; however, if we take $c_{1}=0=\tilde{c}_{1}$ (that is complex instead of almost complex as we had before, leading to $\left.(3.10)\right)^{8}$ then the condition becomes the statement that

$$
\left\langle\tilde{c}_{2} \cdot \frac{1}{3} c_{4}-c_{2} \cdot \frac{1}{3} \tilde{c}_{4},\left[Z^{12}, \partial Z^{12}\right]\right\rangle \in \mathbb{Q} / \mathbb{Z}_{(3)} \cong \mathbb{Z}_{3^{\infty}} \quad \text { has order } 3 .
$$

This can be viewed as our condition on the one-loop term of the topological action reduced to the corner, as in (3.10).

\subsection{Topological modular forms and Tate K-theory}

There are two (related) spectra called Tate K-theory $K_{\text {Tate }}$; one is $k[[q]]$ and another is $K \wedge$ tmf, with a map between them which may be interpreted as a faithfully flat extension in an appropriate category. Laures identifies the K-theory of elliptic cohomology with Katz's universal ring of divided congruences. Tate K-theory is already proposed in [39, 40, 55, 60] to essentially describe the elliptic refinement of the partition function. The point we make here is that the ring of divided congruences is already present in that theory, and hence is of physical significance in the current context.

The ring of divided congruence. The Eisenstein series $E_{4}$ and $E_{6}$ with $E_{2 k}=1-$ $\frac{4 k}{B_{2 k}} \sum_{n=1}^{\infty}\left(\sum_{d \mid n} d^{2 k-1}\right) q^{n}$ generate the graded ring of modular forms over the complex numbers. One can capture congruences between modular forms by considering the ring $D$ where all congruences between modular forms take place. For example, $E_{4} \equiv 1 \bmod 240$, corresponds to the class $\frac{1}{240}\left(E_{4}-1\right) \in D$. However, over the 3 -adic integers, one has to consider the ring $D$ of

\footnotetext{
${ }^{8}$ This conditions natural from the point of view of string theory. Many known compactifications have vanishing first Chern class. Furthermore, the bundles associated to the setting in which the one-loop polynomial arises all satisfy this condition.
} 
divided congruences: the elements of $D$ are those 3 -adically convergent series in $\sum f_{i}$ of (inhomogeneous) modular forms over $\mathbb{Q}_{3}$ such that the $q$-expansion $\sum f_{i}(q)=\lambda\left(\sum f_{i}\right)$ has coefficients $\lambda$ in $\mathbb{Z}_{3}$.

Tate K-theory. Tate K-theory $[2,43]$ is defined via the Tate curve, given by $y^{2}+x y=$ $x^{3}+a_{4} x+a_{6}$, where the coefficients are given by

$$
a_{4}=\frac{1}{48}\left(1-E_{4}\right) \in \mathbb{Z}[[q]], \quad a_{6}=\frac{1}{576}\left(1-E_{4}\right)+\frac{1}{864}\left(E_{6}-1\right) \in \mathbb{Z}[[q]] .
$$

The Tate curve is already defined over the subring $D \subset \mathbb{Z}[[q]]$ of divided congruences. Tate $\mathrm{K}$-theory is simply $K[[q]]$ so that the coefficient ring is $\pi_{0} K_{\text {Tate }}=\mathbb{Z}[[q]]$. At the level of the moduli space of curves, this theory is obtained by working formally in the neighborhood of the $j$-invariant taking the value $j=\infty$. Note that if $q=0$ then $a_{4}=0=a_{6}$. So we see that elements in $D$ correspond to higher order terms beyond the classical term. We interpret this, in the spirit of [55], as considering low values of the coupling constant $q$, so that we view Tate K-theory as a sort of a 'perturbative elliptic cohomology'. The sigma-orientation lifts to a map $M U\langle 6\rangle \rightarrow K[[q]]$ and the invariant $\pi_{*}$ MSpin $\rightarrow \mathbb{Z}[[q]]$ asscoiated to the $\sigma$-prientation on $K_{\text {Tate }}$ is the Witten genus $[2] \varphi_{W}(M) \in \mathbb{Z}[[q]]$. More on the String condition in string theory can be found in [61,72].

Modular forms with respect to the congruence subgroup $\Gamma=\Gamma_{\mathbf{1}}(\mathbf{3})$. Consider the subgroup $\mathrm{SL}\left(2, \mathbb{Z}_{3}\right)=\mathrm{SL}(2, \mathbb{Z}) / \Gamma(3)$ of $\mathrm{SL}(2, \mathbb{Z})$, with the kernel $\Gamma(3)$ a proper subgroup of the congruence subgroup

$$
\Gamma_{1}(3)=\left\{\left(\begin{array}{ll}
1 & * \\
0 & 1
\end{array}\right) \bmod 3\right\} \subset \mathrm{SL}(2, \mathbb{Z}) .
$$

The ring of modular forms over $\Gamma=\Gamma_{1}(3)$ is generated by the two series

$$
E_{1}=1+6 \sum_{n=1}^{\infty} \sum_{d \mid n}\left(\frac{d}{3}\right)_{L} q^{n}, \quad E_{3}=1-9 \sum_{n=1}^{\infty} \sum_{d \mid n}\left(\frac{d}{3}\right)_{L} d^{2} q^{n}
$$

where $\left(\frac{d}{3}\right)_{L}$ denotes the Legendre symbol. Thus in dealing with the $f$-invariant, in addition to the modular forms $E_{4}$ and $E_{6}$, one might encounter the forms $E_{1}$ and $E_{3}$.

\subsection{Chromatic level 2: Heterotic corners and the $f$-invariant}

We have used the $f$-invariant at the level of cohomology classes. We now show that the refined version, the geometric $f$-invariant $[19,83]$, also captures part of the dynamics and anomalies of the heterotic corner.

Elliptic genera and $\langle\mathbf{2}\rangle$-manifolds. The elliptic genera provide interesting invariants for $\langle 2\rangle$-manifolds. Instead of $\mathbb{Q} / \mathbb{Z}$ for the $e$-invariant, one wants to consider some values that 'combine' $\mathbb{Q} / \mathbb{Z}$ with modular forms. For a 12-manifold, this is done by Katz's ring of divided congruences [42]. A framed 10-manifold is the corner of a (U, fr $)^{3}$-manifold if and only if the $f$-invariant gives an integral inhomogeneous modular form for two levels $\geq 2$ which are relatively prime to each other.

Let $X$ be a closed $U$-manifold. Then the elliptic genus of $X$ has an integral $q$-expansion [31]. Let $M^{10}$ be a codimension-two corner of a $\left(U\right.$, fr)-manifold $Z^{12}$. The classical constant term and the quantum nonzero $q$ term are given, respectively, by

$$
\operatorname{Ell}_{0}=\left.\operatorname{Ell}\right|_{q=0}, \quad \widetilde{\operatorname{Ell}}=\text { Ell }- \text { Ell }_{0} .
$$

We consider the splitting of the tangent bundle into two bundles $V_{1}$ and $V_{2}$. Using the relative Chern classes of the split tangent bundle, the $f$-invariant of the framed bordism class of $M^{10}$ is 
defined to be [83]

$$
f\left(M^{10}\right) \equiv\left\langle\left(\operatorname{Ell}\left(V_{1}\right)-1\right)\left(\operatorname{Ell}_{0}\left(V_{2}\right)-1\right),\left[Z^{12}, \partial Z^{12}\right]\right\rangle \bmod \bar{D}_{6}^{\Gamma},
$$

where $\bar{D}_{6}^{\Gamma}$ is described as follows. Denote by $M_{6}^{\Gamma}$ the graded ring of modular forms with respect to $\Gamma=\Gamma_{1}(3)$ which expand integrally, i.e. which lie in $\mathbb{Z}^{\Gamma}[[q]]$. The ring of divided congruences $D_{6}^{\Gamma}$ consists of those rational combinations of modular forms which expand integrally. Then $\bar{D}_{6}^{\Gamma}=$ $D_{6}^{\Gamma}+M_{0}^{\Gamma} \otimes \mathbb{Q}+M_{6}^{\Gamma} \otimes \mathbb{Q}$. Hence $f$ takes values in $\bar{D}_{6}^{\Gamma} \otimes \mathbb{Q} / \mathbb{Z}$, and thus is a natural generalization of the $e$-invariant, which takes values in $\mathbb{Q} / \mathbb{Z}[42]$.

Note that in (3.11) one factor in the integrand is refined while the other factor is classical. This can be viewed as a heterotic analog of the elliptic refinement of the one-loop term in type IIA string theory in [61]. There, only the one-loop polynomial was refined from $I_{8}$ to $I_{8}(q)$, while the $C$-field and its field strength $G_{4}$ remained classical. The end result is that the whole term $G_{4} \wedge I_{8}$ is refined to $q$-expansions.

The geometric $f$-invariant. We now consider a connection on the tangent bundle of the $(U, \mathrm{fr})^{2}$-manifold $Z^{12}$ and hence induced connections $\nabla_{1}$ and $\nabla_{2}$ on the two bundles $V_{1}$ and $V_{2}$. Consider compatible connections, that is ones which preserve trivializations on the faces, i.e. require that they restrict to pure gauge ones on the faces. Let $M^{10}$ be a closed ten-manifold which is a codimension two corner of a $(U, \mathrm{fr})^{2}$-manifold $Z^{12}$ of dimension twelve. Then $M^{10}$ inherits the splitting of its framing. Then, using compatible connections, the geometric $f$ invariant is [83]

$$
\check{f}\left(M^{10},\left.\nabla_{1}\right|_{M},\left.\nabla_{2}\right|_{M}\right) \equiv\left[\int_{Z^{12}} \widetilde{\operatorname{Ell}}\left(\nabla_{1}\right) \operatorname{Ell}_{0}\left(\nabla_{2}\right) \quad \bmod \bar{D}_{6}^{\Gamma}\right] \in \bigoplus_{k=0}^{6} M_{k}^{\Gamma} \otimes \mathbb{R},
$$

where the l.h.s. is defined by considering its residue modulo $\bar{D}_{6}^{\Gamma}$. This expression is congruent to zero $\bmod \bar{D}_{6}^{\Gamma}$ when $Z^{12}$ has an empty corner [83]. We view this as the topological contribution to the effective action at the corner. If $M^{10}$ is the codimension-three corner of a $(U, \mathrm{fr})^{3}$-manifold $W^{13}$ then the $f$-invariant of $M^{10}$ is trivial. Therefore, as indicated earlier, in order to detect nontrivial elliptic cohomology information on $M^{10}$, the manifold $Z^{12}$ itself cannot be a boundary.

Products. We now consider heterotic string theory on product manifolds $M^{10}=X_{1}^{n} \times$ $X_{2}^{10-n}$, viewed as a corner of the 12-dimensional manifold $Z^{12}$ and physically interpret the product formulae of [84]. As explained in the introduction, for framing as well as from the structure of the physical fields, it is natural to consider the factors to be odd-dimensional, with the main example being $S^{3} \times S^{7}$, as a corner of the twelve-manifold $\mathbb{D}^{4} \times \mathbb{D}^{8}$. In this case of the general product the $f$-invariant is determined by the complex $e$-invariant of the factors

$$
\check{f}\left(X_{1}^{n} \times X_{2}^{10-n}\right) \equiv m\left(X_{1}^{n}\right) e_{\mathbb{C}}\left(X_{2}^{10-n}\right) \equiv-m\left(X_{2}^{10-n}\right) e_{\mathbb{C}}\left(X_{1}^{n}\right),
$$

where $m\left(X_{i}\right)$ is any modular form of weight $\left(\operatorname{dim} X_{i}+1\right) / 2$ with respect to the group ${ }^{9} \Gamma=\Gamma_{1}(3)$ such that $m\left(X_{i}\right) \equiv e_{\mathbb{C}}\left(X_{i}\right) \bmod \mathbb{Z}^{\Gamma}[[q]]$. We can have modular forms of weight 2 and 4 by taking the factors to be 3- and 7-dimensional, respectively. This is the correct structure detected in [61] in the following sense. The 11-dimensional one-loop term gets refined only as far as the 8-dimensional one-loop polynomial $I_{8}$ is concerned with the $C$-field part still being classical. As pointed out in [61], one can consider the complimentary point of view where the $C$-field itself is refined while $I_{8}$ remains classical. In the decomposition of the ten-dimensional manifold into a product, we see that we have a physical manifestation of the two formulations in formula (3.12). Note that if one of the factors has a trivial $e$-invariant then the geometric $f$-invariant of the product is congruent to zero.

\footnotetext{
${ }^{9}$ Note that the congruences of (3.11) carry over to arbitrary levels.
} 
Examples. 1. The product $M^{10}=S^{3} \times S^{7}$. Consider the 3 -sphere $S^{3}$ as the sphere bundle of the Hopf line bundle over $S^{2}$. Framing the base and the vertical tangent gives a framing for the total space. The complex $e$-invariant is $e_{\mathbb{C}}\left(S^{3}\right)=-\frac{1}{12}$, while the real $e$-invariant $e_{\mathbb{R}}\left(S^{3}\right)$ is either $-\frac{1}{24}$ or $\frac{11}{24}$, so that $S^{3}$ represents $\nu$. Then $m(\nu)=\frac{1}{12} E_{1}^{2}$, so that $\bar{m}(\nu):=m(\nu)-e_{\mathbb{C}}(\nu)=$ $\frac{1}{12}\left(E_{1}^{2}-1\right)$. The 7 -sphere $S^{7}$, considered as the sphere of the quaternionic line bundle over $S^{4}$, represents $\sigma$. Then $\mathfrak{e}_{\mathbb{C}}(\sigma)=\frac{1}{240}$. One can take $m(\sigma)=\frac{1}{240} E_{4}$ so that $\bar{m}(\sigma):=m(\sigma)-e_{\mathbb{C}}(\sigma)=$ $\frac{1}{240}\left(E_{4}-1\right)$. The geometric $f$-invariant for the product is then [84]

$$
\check{f}\left(S^{3} \times S^{7}\right) \equiv \frac{1}{12 \cdot 240}\left(E_{4}-1\right) .
$$

2. The product $\boldsymbol{M}^{\mathbf{1 0}}=\left(\boldsymbol{S}^{\mathbf{1}}\right)^{\mathbf{3}} \times \boldsymbol{S}^{\mathbf{7}}$. For $\eta \in \pi_{1}^{\mathrm{S}} \cong Z_{2}, e_{\mathbb{C}}(\eta)=\frac{1}{2}$. However, note here that $e_{\mathbb{C}}\left(\eta^{3}\right)=0$. Consequently,

$$
\check{f}\left(\left(S^{1}\right)^{3} \times S^{7}\right) \equiv 0 \cdot \frac{1}{240}\left(E_{4}-1\right) \equiv 0 .
$$

Other products involving combinations of $S^{1} \cong \mathrm{U}(1)$ factors with $S^{3} \cong \mathrm{SU}(2)$ or $S^{7}$ can be treated similarly.

The physical interpretation we provide for these examples of [84] illustrate the reduction to the corner of the topological term of the M-theory effective action is captured by a topological index in twelve dimensions. Since $E_{4}=1+\mathcal{O}(q)$ then the geometric $f$-invariant detects $\mathcal{O}(q)$ information. We hope that this paper helps provide some insight into the role of elliptic cohomology in M-theory, which certainly deserves a better understanding and further investigation. In particular, we hope that the physical setting we provided will help in understanding the $f$-invariant.

Hierarchy of topological theories. The proposals and connections put forth here for M-theory can be applied to the M-branes, i.e. to the branes inside M-theory. Indeed, M-branes with faring and/or corners are studied recently in [69], where tantalizing connections to various topological invariants, including the $f$-invariant, are uncovered. Furthermore, one can study aspects of both M-branes as well as M-theory itself in the context of extended topological quantum field theories (TQFTs). Such theories require corners of increasing codimension all the way up to the maximal, when the theory is fully extended. Our study here concerns, together with [62, 63], point to viewing a sector in M-theory as a 2-extended TQFT. The work [69] also points to a similar description for the M-branes themselves. In all cases one has manifolds of dimension $4 k$ with boundary of dimension $4 k-1$ and codimenison- 2 corner of dimension $4 k-2$, with $k=1,2,3$ for the M2-brane, the M5-brane, and M-theory (spacetime) respectively. The picture that we advocate for the above theories can be captured by the following table

\begin{tabular}{|c|c|c|c|}
\hline Dimension & Type of theory & Boundary/corner structure & Invariants \\
\hline \hline $4 k$ & Topological gauge theory & manifold with corners of codim-2 & primary classes \\
\hline $4 k-1$ & Chern-Simons theory & boundary manifold with boundary & secondary classes \\
\hline $4 k-2$ & Wess-Zumino-Witten theory & corner of codim-2 & tertiary classes \\
\hline
\end{tabular}

The three cases give a ladder of structures of the form 2-3-4, 6-7-8, and 10-11-12. The TQFT aspect of this is studied extensively in [70] within the cobordism hypothesis, with interpretation as ladders of theories of the form $\{$ Topological gauge theory $\}-\{$ Chern-Simons theory $\}-\{$ WessZumino-Witten theory $\}$. One main point to highlight here is that in order for this to work, one changes the interpretation of the forms at the middle stage, i.e. at the boundary Chern-Simons theory, where the form which is an $n$-connection is now interpreted (appropriately) as an $n$ curvature, where the value of the positive integer $n$ depends on the specific theory. 
The top-dimensional case is M-theory itself, with a boundary and a corner, sitting in the following schematic diagrams
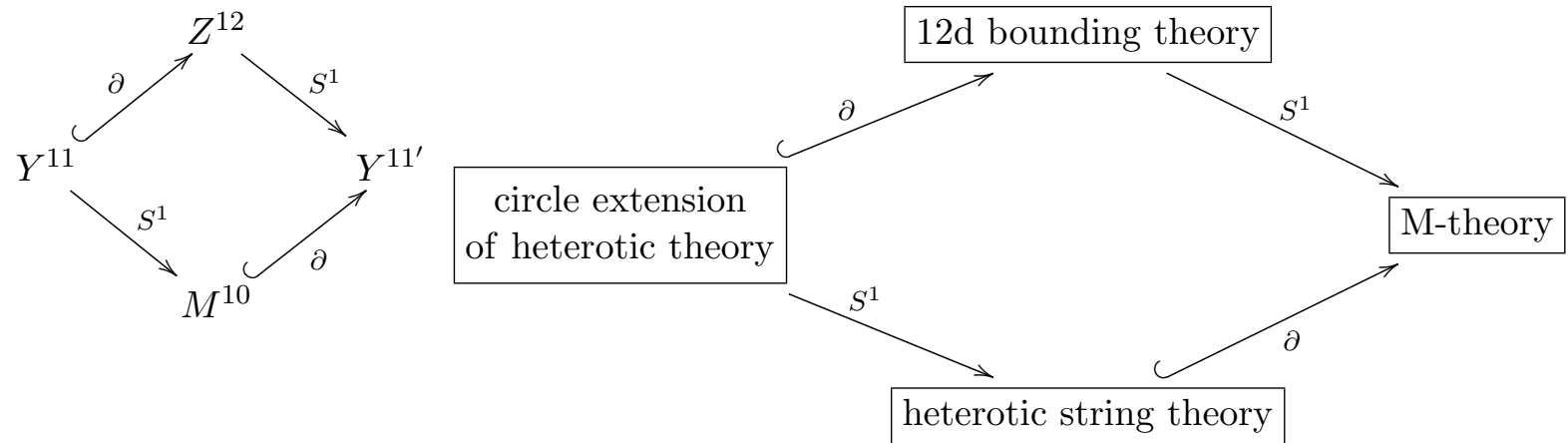

A representative of such a a situation for the case for the M-branes was studied extensively in [69]. The analog in the current setting for the case of M-theory is captured by main example of the product of two closed disks, leading to a two-step reduction of the form

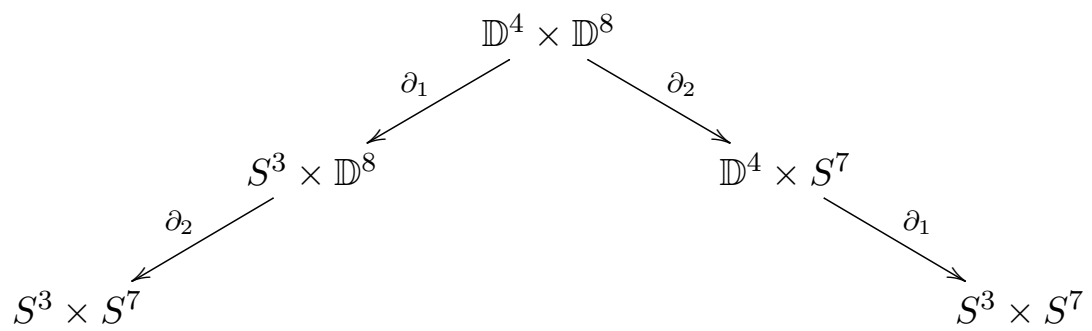

We now can describe this from the point of view of cobordism. We start with the corner $S^{3} \times S^{7}$ and consider it as a boundary in two different ways, namely for $\mathbb{D}^{4} \times S^{7}$ and for $S^{3} \times \mathbb{D}^{8}$. These two are related by a surgery which is implemented by the corresponding space $\mathbb{D}^{4} \times \mathbb{D}^{8}$. The situation is depicted in this diagram

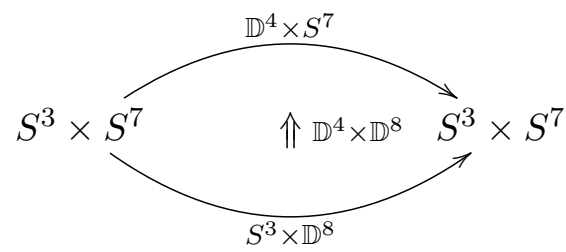

which describes a 2-category of cobordisms, as appropriate in the existence of codimension-2 corners. This deserves more discussion and will be fully developed elsewhere.

The (generalized) WZW theory. The ten-dimensional string theory can be viewed in two ways: First, as a codimension-2 corner and, second, as a (generalized) Wess-Zumino-Witten (WZW) theory. The first is emphasized in this paper and the second is highlighted in previous works $[58,59,61,67]$. Elliptic cohomology on the one hand is used to defined the $f$-invariant, which comes up as the elliptic genus of codimension-2 manifolds [42]. Elliptic cohomology is also used to describe the partition function in the heterotic theory; see [61] for a description in this context. This can also be described via the notion of fibered WZW theory, string-theoretically realizing elliptic genera [23]. In addition to this general conceptual reasoning, we have provided above an explicit relation to the main topological term in the theory, namely the one-loop term, also complementing a similar discussion in [61]. 


\section{Acknowledgements}

The author thanks Ulrich Bunke for explaining his work and Niranjan Ramachandran for discussions on divided congruences. This research is supported by NSF Grant PHY-1102218. The author is indebted to the anonymous referees for many corrections and helpful suggestions.

\section{References}

[1] Aganagic M., Bouchard V., Klemm A., Topological strings and (almost) modular forms, Comm. Math. Phys. 277 (2008), 771-819, hep-th/0607100.

[2] Ando M., Hopkins M.J., Strickland N.P., Elliptic spectra, the Witten genus and the theorem of the cube, Invent. Math. 146 (2001), 595-687.

[3] Astey L., Gitler S., Micha E., Pastor G., Parallelizability of complex projective Stiefel manifolds, Proc. Amer. Math. Soc. 128 (2000), 1527-1530.

[4] Astey L., Guest M.A., Pastor G., Lie groups as framed boundaries, Osaka J. Math. 25 (1988), 891-907.

[5] Atiyah M.F., Patodi V.K., Singer I.M., Spectral asymmetry and Riemannian geometry. I, Math. Proc. Cambridge Philos. Soc. 77 (1975), 43-69.

[6] Atiyah M.F., Singer I.M., Index theory for skew-adjoint Fredholm operators, Inst. Hautes Études Sci. Publ. Math. (1969), 5-26.

[7] Atiyah M.F., Singer I.M., The index of elliptic operators. IV, Ann. of Math. 93 (1971), 119-138.

[8] Atiyah M.F., Singer I.M., The index of elliptic operators. V, Ann. of Math. 93 (1971), 139-149.

[9] Atiyah M.F., Smith L., Compact Lie groups and the stable homotopy of spheres, Topology 13 (1974), $135-142$.

[10] Becker J.C., Schultz R.E., Fixed-point indices and left invariant framings, in Geometric Applications of Homotopy Theory, Vol. I (Proc. Conf., Evanston, Ill., 1977), Lecture Notes in Math., Vol. 657, Springer, Berlin, 1978, 1-31.

[11] Becker K., Becker M., Dasgupta K., Green P.S., Sharpe E., Compactifications of heterotic strings of nonKähler complex manifolds. II, Nuclear Phys. B 678 (2004), 19-100, hep-th/0310058.

[12] Belov D., Moore G.W., Holographic action for the self-dual field, hep-th/0605038.

[13] Bismut J.M., Cheeger J., Families index for manifolds with boundary, superconnections, and cones. I. Families of manifolds with boundary and Dirac operators, J. Funct. Anal. 89 (1990), 313-363.

[14] Borel A., Hirzebruch F., Characteristic classes and homogeneous spaces. III, Amer. J. Math. 82 (1960), 491-504.

[15] Bouwknegt P., Evslin J., Jurčo B., Mathai V., Sati H., Flux compactifications on projective spaces and the S-duality puzzle, Adv. Theor. Math. Phys. 10 (2006), 345-394, hep-th/0501110.

[16] Brasselet J.P., Seade J., Suwa T., Vector fields on singular varieties, Lecture Notes in Math., Vol. 1987, Springer-Verlag, Berlin, 2009.

[17] Bredon G.E., Kosiński A., Vector fields on $\pi$-manifolds, Ann. of Math. 84 (1966), 85-90.

[18] Bunke U., Naumann N., Secondary invariants for String bordism and tmf, arXiv:0912.4875.

[19] Bunke U., Naumann N., The f-invariant and index theory, Manuscripta Math. 132 (2010), 365-397, arXiv:0808.0257.

[20] Čadek M., Crabb M., G-structures on spheres, Proc. London Math. Soc. 93 (2006), 791-816, math.KT/0510149.

[21] Diaconescu E., Moore G., Freed D.S., The M-theory 3-form and $E_{8}$ gauge theory, in Elliptic Cohomology, London Math. Soc. Lecture Note Ser., Vol. 342, Cambridge Univ. Press, Cambridge, 2007, 44-88, hep-th/0312069.

[22] Diaconescu E., Moore G., Witten E., $E_{8}$ gauge theory, and a derivation of K-Theory from M-Theory, Adv. Theor. Math. Phys. 6 (2002), 1031-1134, hep-th/0005090.

[23] Distler J., Sharpe E., Heterotic compactifications with principal bundles for general groups and general levels, Adv. Theor. Math. Phys. 14 (2010), 335-397, hep-th/0701244.

[24] Duff M.J., Liu J.T., Minasian R., Eleven-dimensional origin of string/string duality: a one-loop test, Nuclear Phys. B 452 (1995), 261-282, hep-th/9506126. 
[25] Evslin J., Sati H., Can D-branes wrap nonrepresentable cycles?, J. High Energy Phys. 2006 (2006), no. 10, 050, 10 pages, hep-th/0607045.

[26] Figueroa-O'Farrill J., Kawano T., Yamaguchi S., Parallelisable heterotic backgrounds, J. High Energy Phys. 2003 (2003), no. 10, 012, 22 pages, hep-th/0308141.

[27] Freed D.S., Dirac charge quantization and generalized differential cohomology, in Surveys in Differential Geometry, Surv. Differ. Geom., Vol. VII, Int. Press, Somerville, MA, 2000, 129-194, hep-th/0011220.

[28] Galvez Carrillo M.I., Modular invariants for manifolds with boundary, Ph.D. Thesis, Universitat Autonoma de Barcelona, 2001.

[29] Gran U., Papadopoulos G., Roest D., Supersymmetric heterotic string backgrounds, Phys. Lett. B 656 (2007), 119-126, arXiv:0706.4407.

[30] Gran U., Papadopoulos G., Sloane P., Roest D., Geometry of all supersymmetric type I backgrounds, J. High Energy Phys. 2007 (2007), no. 8, 074, 75 pages, hep-th/0703143.

[31] Hirzebruch F., Berger T., Jung R., Manifolds and modular forms, Aspects of Mathematics, Vol. E20, Friedr. Vieweg \& Sohn, Braunschweig, 1992.

[32] Hopkins M.J., Singer I.M., Quadratic functions in geometry, topology, and M-theory, J. Differential Geom. 70 (2005), 329-452, math.AT/0211216.

[33] Kamata M., Minami H., The special orthogonal groups $\mathrm{SO}(2 n)$ as framed boundaries, Kyushu J. Math. 54 (2000), 147-153.

[34] Kawano T., Yamaguchi S., Dilatonic parallelizable NS-NS backgrounds, Phys. Lett. B 568 (2003), 78-82, hep-th/0306038.

[35] Kervaire M., Courbure intégrale généralisée et homotopie, Math. Ann. 131 (1956), 219-252.

[36] Kervaire M.A., Milnor J.W., Groups of homotopy spheres. I, Ann. of Math. 77 (1963), 504-537.

[37] Killingback T.P., Global anomalies, string theory and spacetime topology, Classical Quantum Gravity 5 (1988), 1169-1185.

[38] Knapp K., Rank and Adams filtration of a Lie group, Topology 17 (1978), 41-52.

[39] Kriz I., Sati H., M-theory, type IIA superstrings, and elliptic cohomology, Adv. Theor. Math. Phys. 8 (2004), 345-394, hep-th/0404013.

[40] Kriz I., Sati H., Type II string theory and modularity, J. High Energy Phys. 2005 (2005), no. 8, 038, 30 pages, hep-th/0501060.

[41] Kriz I., Sati H., Type IIB string theory, S-duality, and generalized cohomology, Nuclear Phys. B 715 (2005), 639-664, hep-th/0410293.

[42] Laures G., On cobordism of manifolds with corners, Trans. Amer. Math. Soc. 352 (2000), 5667-5688.

[43] Laures G., K(1)-local topological modular forms, Invent. Math. 157 (2004), 371-403.

[44] Lerche W., Nilsson B.E.W., Schellekens A.N., Warner N.P., Anomaly cancelling terms from the elliptic genus, Nuclear Phys. B 299 (1988), 91-116.

[45] Löffler P., Smith L., Line bundles over framed manifolds, Math. Z. 138 (1974), 35-52.

[46] Mahowald M., Rezk C., Topological modular forms of level 3, Pure Appl. Math. Q. 5 (2009), 853-872, arXiv:0812.2009.

[47] Maldacena J., Moore G., Seiberg N., D-brane instantons and K-theory charges, J. High Energy Phys. 2001 (2001), no. 11, 062, 42 pages, hep-th/0108100.

[48] Mathai V., Sati H., Some relations between twisted $K$-theory and $E_{8}$ gauge theory, J. High Energy Phys. 2004 (2004), no. 3, 016, 22 pages, hep-th/0312033.

[49] Melrose R.B., Piazza P., An index theorem for families of Dirac operators on odd-dimensional manifolds with boundary, J. Differential Geom. 46 (1997), 287-334.

[50] Minami H., Stiefel manifolds as framed boundaries, Osaka J. Math. 27 (1990), 185-189.

[51] Minami H., Remarks on framed bordism classes of classical Lie groups, Publ. Res. Inst. Math. Sci. 43 (2007), 461-470.

[52] Moore G., Saulina N., T-duality, and the $K$-theoretic partition function of type IIA superstring theory, Nuclear Phys. B 670 (2003), 27-89, hep-th/0206092.

[53] Pittie H.V., Smith L., Generalized flag manifolds as framed boundaries, Math. Z. 142 (1975), $191-193$.

[54] Sadri D., Sheikh-Jabbari M.M., String theory on parallelizable pp-waves, J. High Energy Phys. 2003 (2003), no. 6, 005, 35 pages, hep-th/0304169. 
[55] Sati H., The elliptic curves in gauge theory, string theory, and cohomology, J. High Energy Phys. 2006 (2006), no. 3, 096, 20 pages, hep-th/0511087.

[56] Sati H., An approach to anomalies in M-theory via KSpin, J. Geom. Phys. 58 (2008), 387-401, arXiv:0705.3484.

[57] Sati H., $\mathbb{O P}^{2}$ bundles in M-theory, Commun. Number Theory Phys. 3 (2009), 495-530, arXiv:0807.4899.

[58] Sati H., The loop group of $E_{8}$ and targets for spacetime, Modern Phys. Lett. A 24 (2009), 25-40, hepth/0701231.

[59] Sati H., $E_{8}$ gauge theory and gerbes in string theory, Adv. Theor. Math. Phys. 14 (2010), 399-437, hep-th/0608190.

[60] Sati H., Geometric and topological structures related to M-branes, in Superstrings, Geometry, Topology, and $C^{*}$-Algebras, Proc. Sympos. Pure Math., Vol. 81, Amer. Math. Soc., Providence, RI, 2010, 181-236, arXiv:1001.5020.

[61] Sati H., Anomalies of $E_{8}$ gauge theory on string manifolds, Internat. J. Modern Phys. A 26 (2011), 21772197, arXiv:0807.4940.

[62] Sati H., Constraints on heterotic M-theory from s-cobordism, Nuclear Phys. B 853 (2011), 739-759, arXiv:1102.1171.

[63] Sati H., Corners in M-theory, J. Phys. A: Math. Theor. 44 (2011), 255402, 21 pages, arXiv:1101.2793.

[64] Sati H., M-theory, the signature theorem, and geometric invariants, Phys. Rev. D 83 (2011), 126010, 10 pages, arXiv:1012.1300.

[65] Sati H., On global anomalies in type IIB string theory, arXiv:1109.4385.

[66] Sati H., Topological aspects of the partition function of the NS5-brane, arXiv:1109.4834.

[67] Sati H., Duality and cohomology in M-theory with boundary, J. Geom. Phys. 62 (2012), 1284-1297, arXiv:1012.4495.

[68] Sati H., Geometry of Spin and $\operatorname{Spin}^{c}$ structures in the M-theory partition function, Rev. Math. Phys. 24 (2012), 1250005, 112 pages, arXiv:1005.1700.

[69] Sati H., Framed M-branes, corners, and topological invariants, arXiv:1310.1060.

[70] Sati H., String theory as local pre-quantum field theory, in preparation.

[71] Sati H., Schreiber U., Stasheff J., Fivebrane structures, Rev. Math. Phys. 21 (2009), 1197-1240, arXiv:0805.0564.

[72] Sati H., Schreiber U., Stasheff J., Twisted differential string and fivebrane structures, Comm. Math. Phys. 315 (2012), 169-213, arXiv:0910.4001.

[73] Sati H., Westerland C., Twisted Morava K-theory and E-theory, arXiv:1109.3867.

[74] Schellekens A.N., Warner N.P., Anomalies, characters and strings, Nuclear Phys. B 287 (1987), 317-361.

[75] Singhof W., Parallelizability of homogeneous spaces. I, Math. Ann. 260 (1982), 101-116.

[76] Singhof W., The $d$-invariant of compact nilmanifolds, Invent. Math. 78 (1984), 113-115.

[77] Singhof W., Wemmer D., Parallelizability of homogeneous spaces. II, Math. Ann. 274 (1986), $157-176$.

[78] Smith L., Framings of sphere bundles over spheres, the plumbing pairing, and the framed bordism classes of rank two simple Lie groups, Topology 13 (1974), 401-415.

[79] Spindel P., Sevrin A., Troost W., Van Proeyen A., Complex structures on parallelised group manifolds and supersymmetric $\sigma$-models, Phys. Lett. B 206 (1988), 71-74.

[80] Steer B., Orbits and the homotopy class of a compactification of a classical map, Topology 15 (1976), 383-393.

[81] Stong R.E., Notes on cobordism theory, Mathematical Notes, Princeton University Press, Princeton, N.J., 1968.

[82] Thomas E., Cross-sections of stably equivalent vector bundles, Quart. J. Math. Oxford Ser. (2) 17 (1966), 53-57.

[83] von Bodecker H., On the geometry of the $f$-invariant, arXiv:0808.0428.

[84] von Bodecker H., On the $f$-invariant of products, arXiv:0909.3968.

[85] Witten E., The index of the Dirac operator in loop space, in Elliptic Curves and Modular Forms in Algebraic Topology (Princeton, NJ, 1986), Lecture Notes in Math., Vol. 1326, Springer, Berlin, 1988, 161-181.

[86] Witten E., On flux quantization in $M$-theory and the effective action, J. Geom. Phys. 22 (1997), 1-13, hep-th/9609122.

[87] Wood R.M.W., Framing the exceptional Lie group G2, Topology 15 (1976), 303-320. 